\title{
AM FUßE DES VULKANS - ORGELKUNST UND KONTRAPUNKT IN NEAPEL UM 1600
}

\author{
von JOHANNES RING
}

\section{Zusammenfassung:}

1575 veröffentlichte Rocco Rodio in Neapel in seinem Libro di ricercate eine Fantasia über den Kirchenhymnus "Ave maris stella". Im Kontext der progressiven Beiträge zur Tastenmusik seiner italienischen Zeitgenossen Antonio Valente (1575), Claudio Merulo und Andrea Gabrieli erscheint Rodios Cantus firmus-Satz geradezu anachronistisch. Doch Rodios Intention ist die des Kontrapunktikers. Für ihn war die kontrapunktische Kunst die entscheidende Basis des kompositorischen Handwerkes. Rodios Intention wird beleuchtet durch das Umfeld seines Wirkens -den diskussionsfreudigen Akademien in Neapel- und durch Ereignisse in Rom und Palermo, die im Auftritt des Spaniers Sebastián Raval gipfeln. Ihren eigentlichen musikhistorischen Kontext findet Rodios „Ave maris stella“ in der (liturgischen) Orgelmusik von Antonio de Cabezón, Manuel Rodriguez Coelho und Jean Titelouze und vielleicht sogar von António Carreira.

\begin{abstract}
(At the Foot of the Volcano - Organ and Counterpoint in Naples around 1600).

In 1575, Rocco Rodio published a "Fantasia" on the hymnus "Ave maris stella" in Naples in his Libro di ricercate. In the context of the progressive contributions to music for keyboard instruments of his contemporaries Antonio Valente (1575), Claudio Merulo and Andrea Gabrieli, Rodio's setting of cantus firmus seems nothing short of anachronistic. But Rodio's intention lies in the implementation of counterpoint. For him, the art of counterpoint was the decisive basis of the composer's craft. Rodio's intention is illuminated by the circumstances of his creative activity -the academies in Naples, always eager to dispute,- and by certain events in Rome and Palermo that culminated in the appearance of the Spaniard named Sebastián Raval.

The original context regarding the history of music for Rodio's "Ave maris stella" is in the liturgical organ music by Antonio de Cabezón, Manuel Rodriguez Coelho und Jean Titelouze und perhaps by António Carreira.
\end{abstract}

\section{Introduzione - una fantasia capricciosa}

Signore Rodio und Seminarista Emilio stiegen die enge Steintreppe der Orgelempore hinab. Vor dem Kirchenportal entlohnte Rocco Rodio den jungen Emilio, der ihm während der vergangenen Stunde die Bälge getreten hatte. Anschließend ging Rodio nicht wie so oft in die Camerata di Propaganda per l'Affinamento del Gusto Musicale, um mit Kollegen zu diskutieren und das neue schwarze Bohnengetränk (Coffea canephora) zu genießen, das sich der spanische Vizekönig von portugiesischen Händlern liefern ließ. Wie so manchmal zog es der maestro vor, einen Spaziergang durch die schöne und sonnendurchflutete Landschaft mit Blick auf den Golf und den Vulkan zu unternehmen. Auf seinem Spaziergang wollte er weiter darüber sinnieren, wie er zu einer kunstvollen und pragmati- 
schen Synthese aus seinen anspruchsvollen kontrapunktischen Eingebungen und den spieltechnischen Möglichkeiten der Tastenmusik gelangen könnte.

I.

Diese stimmungsvolle kleine Fiktion mag als Einleitung zur Beschäftigung mit einer bemerkenswerten Orgelmusik aus Italien dienen. Der Name Rocco Rodio (ca. 1535-1615) ist den heutigen Organisten bzw. Tastenmusikern kaum bekannt - die Musikforschung hat in verschiedenen kleineren Arbeiten bzw. in Übersichtswerken auf ihn hingewiesen, z.B. Joseph A. Burns, Willi Apel und zusammenfassend in einem Lexikonartikel Josef-Horst Lederer ${ }^{1}$. Macário Santiago Kastner und James Dalton $^{2}$ wurden seiner Bedeutung durch heute leider nur wenig bekannte (Teil-) Editionen seiner Tastenmusik gerecht. Neue Impulse erhielt die Rodioforschung durch Dinko Fabris und Diego Cannizzaro $^{3}$. Sie haben Rodio aus der archivalen Versenkung geholt, seinen Namen und sein Wirken in Erinnerung zurückgerufen und einen Teil seines Werkes der Öffentlichkeit zugänglich gemacht.

Thema der folgenden Betrachtungen ist eine Komposition über „Ave maris stella“, ein auf den ersten Blick sperrig wirkender und kaum praktizierbarer Satz ${ }^{4}$, den Rodio in seinem Libro di ricercate a quattro voci con alcune fantasie sopra varii canti fermi novamente posti in luce 1575 in Neapel publizierte $^{5}$. Rodios akademisch wirkende Fantasie über „Ave maris stella“ scheint im Kontext der progressiven Beiträge zur Tastenmusik seiner Zeitgenossen - besonders Claudio Merulo und Andrea Gabrieli in Venedig und vor Ort Antonio Valente - geradezu anachronistisch. Ob es sich tatsächlich so verhält, sollen die nächsten Seiten zeigen.

1. Arthur Mendel, Pitch in the 16th and Early 17th Centuries - Part IV, in: Musical Quarterly, Vol. 34, No. 4 (1948), S. 575-93. Joseph Albert Burns, Antonio Valente, Neapolitan Keyboard Primitive, in: Journal of American Musicological Society, Vol. XII (1959), S. 133-43 [= Burns]. W. Apel, Die süditalienische Clavierschule des 17. Jahrhunderts, in: Acta Musicologica, XXXIV (1962), S. 128ff. J.-H. Lederer, Rodio, Rocco, (art.) in: The New Grove, Dictionary of Music \& Musicians [= NGroveD], hrsg. von Stanley Sadie, Vol. 16, London 1980, S. 91 [= Lederer]. Zumindest erwähnt wird Rodio in: Hans Klotz, Über die Orgelkunst der Gotik, der Renaissance und des Barocks, Kassel u.a., 1975. S. 131. [= Klotz], in: Gustave Reese, Music In The Renaissance, rev. ed. New York 1959. S. 486 \& 532 [= Reese], in: Dagmar Teepe, Die Entwicklung der Fantasie für Tasteninstrumente im 16. und 17. Jahrhundert, [Kieler Schriften zur Musikwissenschaft XXXVI], Kassel u.a. 1990, in gattungsformalen Lexikonartikeln zur Fantasia und dem Ricercare: Christopher D. S. Field, Fantasia (art.) in: NGroveD Vol. 6, S. 382 [= Field] und John Caldwell, Ricercare (art.) in: NGroveD Vol. 15, S. 837 [= Caldwell].

2. Rocco Rodio, Cinque Ricercate, una fantasia per organo, clavicembalo, clavicordo o arpa, hrsg. von Macario Santiago Kastner, Padua, 1958. Faber Early Organ Series, European Organ Music Of The Sixteenth \& Seventeenth Century, Vol. 16, Italy c.1517-1599. Antico · M.A. Cavazzoni · G. Gavazzoni · Rodio · Valente - Bertoldo · A. Gabrieli $\cdot$ Merulo $\cdot$ Luzzaschi $\cdot$ Pellegrini, London 1988, S. 14/15. [= Italy c.1517-1599]. Die Notenbeispiele meiner Arbeit basieren auf dieser Ausgabe.

3. D. Fabris, Introduzione ai Salmi per $i$ Vespri di Rocco Rodio, Lamezia Terme, A.M.A. Calabria, 1994. D. Cannizaro, La musica per organo e clavicembalo nei regni di Napoli e di Sicilia tra XVI e XVII secolo, Vol. I. \& II. (Editionen), Rom 2004. Internetpublikation (Pdf). [= Cannizzaro] D. Fabris verfasste auch den Artikel in Musik in Geschichte und Gegenwart, 2. neubearbeitete Ausgabe hrsg. von Ludwig Finscher, Personenteil Band 14, Kassel u.a. 2005, Sp. 249-50. Die meisten verwertbaren Informationen enthält allerdings M. A. Kastners Artikel Rodio, Rocco, in: Die Musik in Geschichte und Gegenwart, hrsg. von Friedrich Blume, Band 11, Kassel u.a. 1963, Sp. 598-600. [= Kastner1963]

4. Willi Apel, Geschichte der Orgel- und Klaviermusik bis 1700, Kassel u.a. 1967 [= Apel1967], S. 120.

5. RISM: A/I R1821. 
II.

Neapel war nach Venedig und Rom ab dem 16. Jahrhundert ein musikalisches Zentrum. Namhafte Komponisten wie Giovanni Maria Trabaci6 (ca. 1575-1647), Organist der Kapelle des spanischen Vizekönigs, verliehen der Hafenstadt internationale Bedeutung. Kapellmeister der königlichen Kapelle in Neapel war der Flame Giovanni de Macque (?1548-1614). Trabaci war de Macques Nachfolger im Amt des Kapellmeisters. Zweiter Organist des Vizekönigs war Ascanio Mayone (ca. 1565-1627). Neben der herausragend besetzten königlichen Kapelle prägten die Bruderschaften das musikalische Leben der Stadt, voran die des Oratorio dei Filippini, wo Trabaci zusätzlich zeitweise Organist war.

Wichtig für die frühe Tastenmusik ist Rodios Zeitgenosse Antonio Valente ${ }^{7}$, der blinde Organist der Kirche San Angelo a Nido (auch Nilo). Valentes Intavolatura de cimbalo: Recercate, fantasie et canzoni francese desminuite: con alcuni tenori balli et varie sorte de contraponti. Libro primo $(1575 / 76)^{8}$ und Rodios Libro di ricercate (1575) sind die frühesten gedruckten Bücher der neapolitanischen Schule von Tastenmusikern.

Beschreiben wir das musikalische Treiben in Neapel, so dürfen die Akademien nicht vergessen werden: Eine der musikalischen Akademien war vom Fürsten Don Fabrizio, dem Vater des berühmten Komponisten Don Carlo Gesualdo, gegründet worden ${ }^{9}$. Von seinem Vater hatte Don Carlo das Mäzenatentum der Musiker um Signore Rodio übernommen, der Mitglied Gesualdos legendären Akademie war ${ }^{10}$. Zusammen mit anderen Musikern hatte Rodio die eingangs erwähnte Camerata di Propaganda per l'Affinamento del Gusto Musicale gegründet, vermutlich als privates Diskussionsforum.

Mit der Konzentration von Musikern und Komponisten mit hoher Reputation, seinen Akademien und musikpädagogischen Institutionen und dem Zusammentreffen von italienischer, spanischer und flämischer Musik war Neapel ein fruchtbarer Boden für intensiven Gedankenaustausch und anspruchsvolle Diskussionen über das Wesen und die Regeln der Musik. Bemerkenswert ist eine ganze Reihe musiktheoretischer Abhandlungen, die im Neapel des 16. und frühen 17. Jahrhunderts veröffentlicht wurden. Mit seinen Regole di musica... tutti i canoni sopra il canto fermo (1600) befindet sich Rodio in guter Gesellschaft ${ }^{11}$ : Luigi Dentice publizierte 1552 seine Duo dialoghi della musica; Scipione

6. Roland Jackson, Trabaci, Giovanni Maria (art.) in: NGroveD, Vol. 19, London 1980, S. 106/07. Faber Early Organ Series, European Organ Music Of The Sixteenth \& Seventeenth Century, Vol. 17, Italy c.1600-1635. E. Pasquini · De Macque . Mayone $\cdot$ Trabaci $\cdot$ Cima $\cdot$ Diruta $\cdot$ Banchieri $\cdot$ Frescobaldi, London 1988, S. VII ff.

7. Roland Jackson, Valente, Antonio, (art.) in: NGroveD, Vol. 19, London 1980, S. 492/93.

8. RISM: A/I V33. S. 86.

9. Reese, S. 430. Claudio Gallico, La Epoca del Humanismo y del Renacimiento, [Historia de la Musica, 4], Madrid 1986,

10. 1573 erschienen in Neapel Rodios Salmi per $i$ Vespri a quattro voci. Der Schluss liegt nahe, dass sich der Komponist mit dieser Publikation der musikalischen Öffentlichkeit seiner neuen Wahlheimat empfehlen wollte.

11. Rodios Traktat ist ediert in: Regole di musica, Bibliotheca Musica Bononiensis, II/56, Bologna 1981 und in: Regole di musica (1609): a machine-readable edition, von Frans Wiering, Utrecht University 2000. Editionen jeweils nach der zweiten Auflage, die Rodios Schüler G. B. Olifante als Anhang in seinem Trattado di Proportioni necessario à detto Libro veröffentlichte, 1609 von Giacomo Carlino und Costantino Vitale in Neapel gedruckt. Siehe Ernst Apfel, Geschichte der Kompositionslehre. Von den Anfängen bis gegen 1700, Teil III: Die Kontrapunkt- und Kompositionslehre nach G. Zarlino in Italien, Deutschland, Frankreich und den übrigen Ländern, Wilhelmshaven 1981, S. 358 [= Apfel]. Nur eine Auswahl weiterer Traktate sei hier aufgeführt. Vergl. Renato Di Benedetto, Naples (It. Napoli) (art.) in: NGroveD, Vol. 13, London 1980, S. 22 - 32, Apfel, S. 357ff. \& 37383 und mit den biografischen Artikeln in NGroveD. 
Cerrotos Della prattica musica vocale et strumentale erschien 1601 und Pietro Cerones El melopeo y maestro, Tractado de Musica teórica y prática 1613. Der Trattado de glosas des berühmten spanischen Komponisten Diego Ortiz, der von 1553 bis zu seinem Tod im Jahr 1570 in Neapel lebte und die meiste Zeit als maestro de capilla der spanischen Vizekönige wirkte, wurde allerdings in Rom gedruckt ${ }^{12}$.

III.

Weniger Rodios dafür aber Valentes Clavierbücher sind frühe Beispiele der Opera omnia. Gemeint sind Sammlungen, die weitgehend die jeweils aktuellen Formen der Tastenmusik -liturgische Kompositionen, Fantasien, Ricercari, Intabulationen, Variationen und Tänze- enthalten. Frühere Beispiele sind Girolamo Cavazzonis Intabulatura Daorgano, cioe misse himni magnificat... (Venedig, 1549) und das Libro de Cifra nueva / para tecla, harpa, y vihuela, ... von Luis Venegas de Henestrosa (Alcalá de Henares, 1557). Allerdings reicht das Libro di ricercate Rodios nicht an die Formenvielfalt Cavazzonis und des Spaniers Venegas de Henestrosa heran. Der Begriff musikalischer Apparat lässt sich synonym zu Opera omnia verwenden.

Rodios und Valentes Drucke haben eine auffällige Gemeinsamkeit, enthalten doch beide eine Komposition über „Salve Regina“. Valentes „Salve Regina“ hat J. Burns treffend charakterisiert: „Sie führt die Choralmelodie in ganzen Noten in einer der mittleren Stimmen durch, während die drei anderen Stimmen als improvisierendes Geflecht gewoben sind."13 Über Valentes Intention, ein einzelnes Stück mit sakraler Thematik in seine Intavolatura de cimbalo aufzunehmen, vermutet Burns, dass Valente der Öffentlichkeit zeigen wollte, dass er in der Lage ist, (ansatzweise) polyphone Sätze (Burns: ,pedantic fantasias“) über einen bekannten Cantus firmus zu schreiben. Ein weiterer Beweggrund für diesen Fremdkörper in einem eher dem cimbalo zugedachten Buch Valentes mag sein, dass durch eine liturgisch gebundene Komposition der Marktwert des Clavierbuchs steigt. Eine größere Käuferschaft wird angesprochen, ein wirtschaftlicher Aspekt bei der Publikation der Opera omnia. Das kontrapunktische und ansatzweise akademisch wirkende Stück wird im wahrsten Sinn des Wortes zu einem Empfehlungsschreiben. Die Melodie der Marienantiphon wird als Cantus planus in Breven durchgeführt. Die drei anderen Stimmen bilden einen mitunter sehr lebhaften Kontrapunkt. Stellenweise kündet das Figurenwerk komplexe Toccatentechnik an, wie sie im 17. Jahrhundert gang und gäbe wurde.

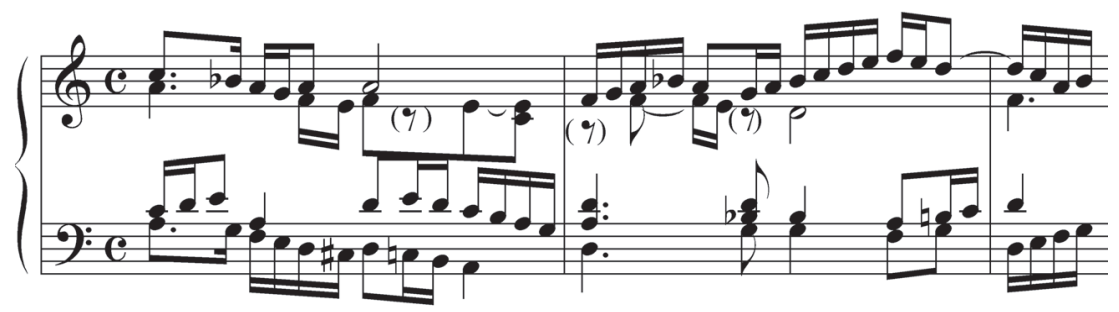

12. Trattado de glosas sobre clausulas y otros generos de puntos en la musica de violones (1553); simultan in Italienisch publiziert als Glose sopra le cadenze et altre sorte de punti in la musica del violone.

13. Vergl. Burns, S. 136.

14. Vergl. Apel1967, S. 121. 
Valente, Takt $21 \mathrm{ff} .{ }^{14}$

Rodio nennt seine vier Cantus firmus-gebundenen Stücke im Titel des Druckes fantasie sopra varii canti fermi. Es sind Fantasien über die schon angesprochene Marienantiphon „Salve Regina“, über die Kirchenhymnen „Iste confessor“ und „Ave maris stella“ und über den legendären SpagnaTon.

Rodios Traktat Regole di musica führt im Titel „canoni sopra il canto fermo“ auf. Genau hier schließt sich der Kreis aus dem Theorietraktat von 1600 und dem Libro di ricercate (1575): Rodios Anliegen war die kontrapunktische Kunst. Seine Ricercari, aber noch viel mehr seine fantasie sopra varii canti fermi sind stimmig komponierte, imitatorische (,canoni“) Sätze. Die fantasie sopra „Salve Regina“" ist von anderer Machart als Valentes Komposition über die Antiphon - bei Rodio dominiert die kontrapunktische Ordnung und nicht das improvisierende Stimmengeflecht wie bei Valente, das ganz von den griffpraktischen Möglichkeiten des Cembalospiels bestimmt ist. Der Anfang von Rodios

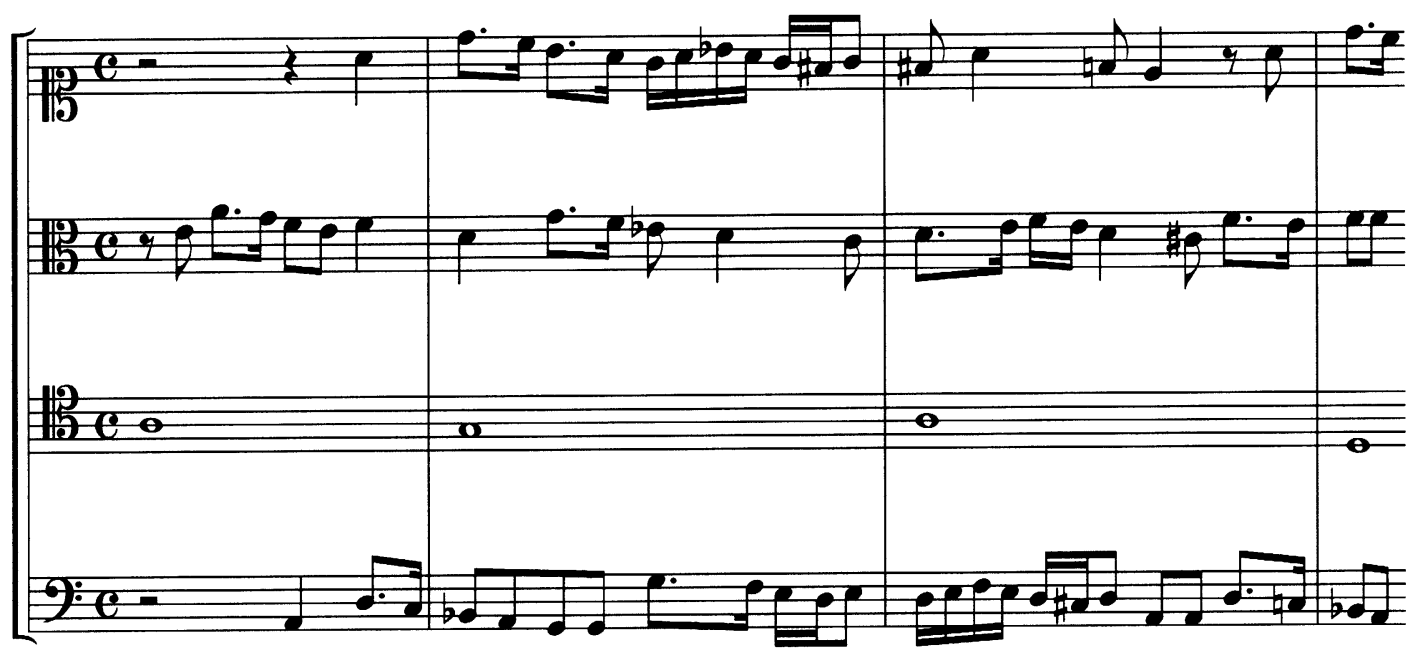

Fantasia über „Salve Regina“::15

In dichten Imitationspunkten sind Rodios kontrapunktische Stimmen miteinander verflochten, während die langen Töne des cantus firmus den Tenor konsequent bestimmen. Die kontrapunktischen Stimmen sind anspruchsvoll und abwechslungsreich gestaltet, so dass der Tastenmusiker durchweg gefordert ist, ähnlich wie in Valentes „Toccatensatz“.

Ebenso arbeitet Rodio seine Fantasia sopra „Ave maris stella“ aus. Melodische Grundlage ist der Kirchenhymnus im 1. Ton.

15. Ebenda, S. 119. 

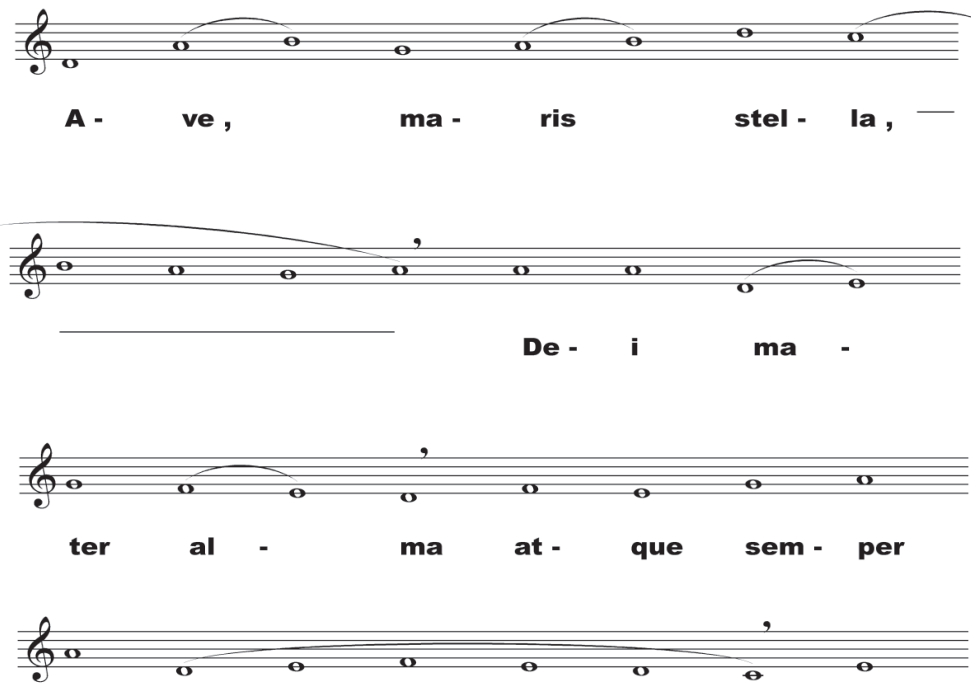

vir - go,

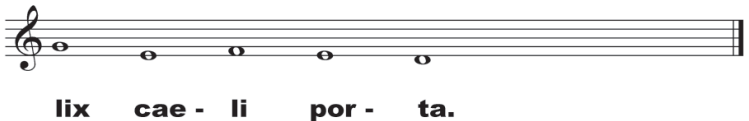

Hymnus „Ave maris stella“, 1. Vers:

Wieder liegt der Canto fermo (= Cantus firmus) als Cantus planus im Tenor (C4- bzw. Tenorschlüssel). Die Hymnusmelodie wird ohne Unterbrechung vom 1. bis zum letzten Takt durchgeführt. Die übrigen drei Stimmen - Discantus / Sopran (C1-Schlüssel), Altus (C3-Schlüssel) und Bassus (F4-Schlüssel) bilden ein oft sehr dichtes kontrapunktisches Geflecht. Die drei kontrapunktischen Stimmen greifen die Tonfolgen des cantus firmus nicht auf. Sie beziehen sich aber stellenweise auf-

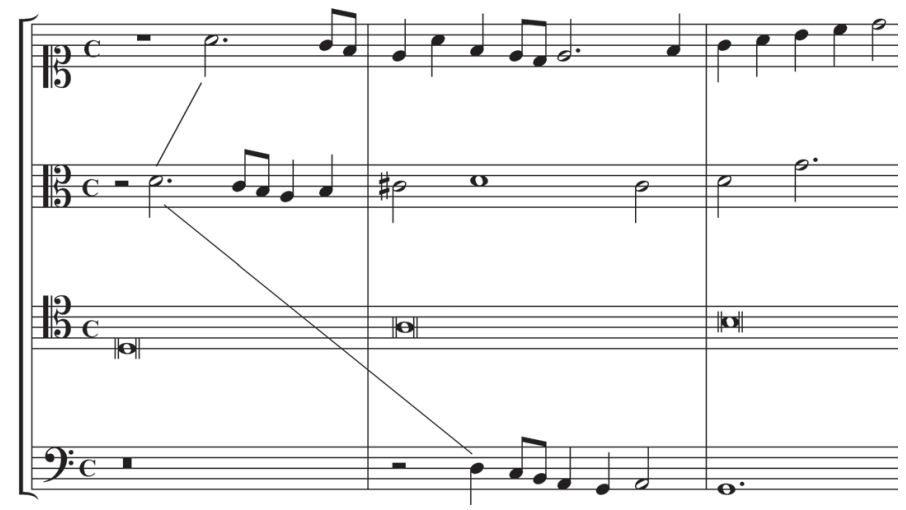


einander. Kleine Imitationspunkte strukturieren den dichten Satz.

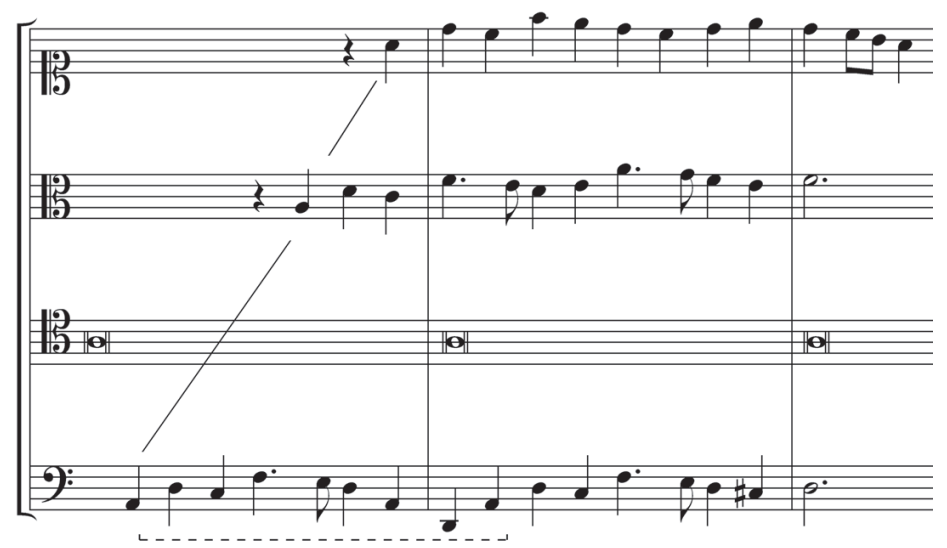

Ab Takt 13 können wir den zweiten Imitationspunkt feststellen.

Das Etablieren neuer Imitationspunkte folgt der textlichen Struktur des Hymnus. Die Notenbeispiele zeigen, wie der Komponist im Zusammenhang mit den textlichen und melodischen Zäsuren neue ,freie“ soggetti einführt.

Anfang „Ave“:

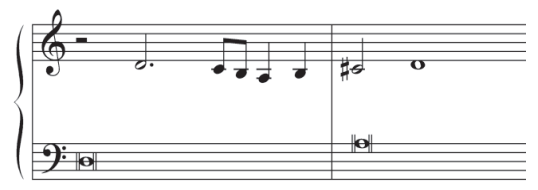

1. Zäsur „stella, Dei“:

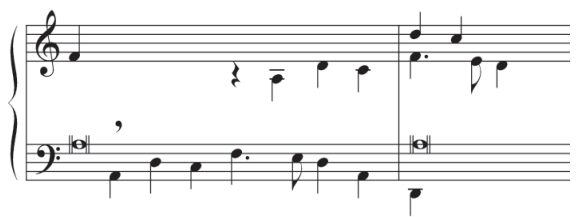

2. Zäsur ,alma atque“:

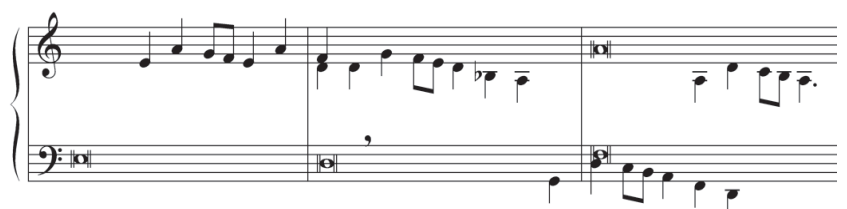

3. Zäsur „felix...":

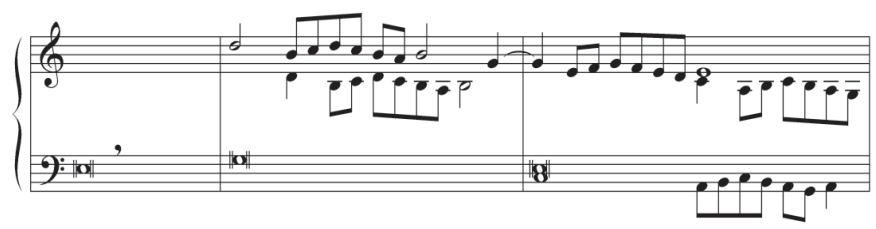


Diese Methode der Durchführung jeder Melodiezeile von Hymnen, Cantica und anderen liturgischen Melodien begegnet uns schon früher, beispielsweise in der oben erwähnten Intabulatura Daorgano Girolamo Cavazzonis, in dem gewaltigen handschriftlichen Konvolut von Jan z Lublina aus Polen ${ }^{16}$, in Juan Bermudos Comiença el libro llamado Declaración de instrumentos musicales..., (Osuna 1555) und in Antonio de Cabezóns posthum erschienenen Obras de Música para Tecla, Arpa y Vihuela,..., (Madrid 1578).

IV.

Anders als die späteren cantus firmus-gebundenen Kompositionen in Form imitatorischer Tentos von Manuel Rodriguez Coelho bzw. „Choral“-Ricercari von Samuel Scheidt, beziehen sich die soggetti in Rodios Fantasien nicht auf den Hymnuston. Im Vergleich zu den choralgebundenen Kompositionen in den soeben aufgezählten Quellen von Bermudo, de Cabezón und Cavazzoni sind Rodios fantasie sopra varii canti fermi schon optisch auffällig: Sein Kontrapunkt verdichtet sich meistens 8 : 1, mitunter sogar in allen drei freien Stimmen. Doch geht Rodio klug mit der komplexen Materie um - einige Passagen sind ruhiger gestaltet. Der dichte Satz in Rodios fantasie sopra varii canti fermi erinnert an die madrigali a note nere. Dunkel gefärbte Notenzeichen, die für kleine Notenwerte stehen und welche die Sätze sehr lebhaft bis hin zu virtuos gestalten, wurden ab Mitte des 16. Jahrhunderts ein gängiges Kompositionsmittel nicht nur süditalienischer Madrigale, so z.B. in Giovanni Continos „Dolce mio ben"17.

Wahrscheinlich wurde dieser Kompositionsstil auch in den Akademien Neapels diskutiert. Später finden wir diese Technik in Madrigalen von Don Carlo Gesualdos, der die Technik der note nere auch in seine geistliche Musik übertrug, wie sich gut in den Responsoria Sanctae Spectantia Et Alia Ad Officium Hebdomadae (Gesualdo [bei Neapel] 1611) beobachten lässt ${ }^{18}$.

16. Kraków, Biblioteka Polsiej Akademii Nauk, 1716.

17. Il vero terzo libro de diversi autori a nota negre [= a note nere], Venedig 1549. Siehe Giovanni Contino, Madrigali A Quattro E Cinque Voci in antologie ed intavolature, hrsg. von Romano Vettori, Mailand 1994. S. 1-7. Siehe auch Don Harran (ed.), The Antologies of Blacknote madrigals, American Institute of Musicology, Stuttgart- Nordhausen 1978-80. [= Corpus Mensurabilis Musicae, 73.]

18. Aus dem 6. Responsorium des Gründonnerstags: Gesualdo Di Venosa, Sämtliche Werke: Responsorien, hrsg. von Glenn E. Watkins, Leipzig 1987, S. 26. 


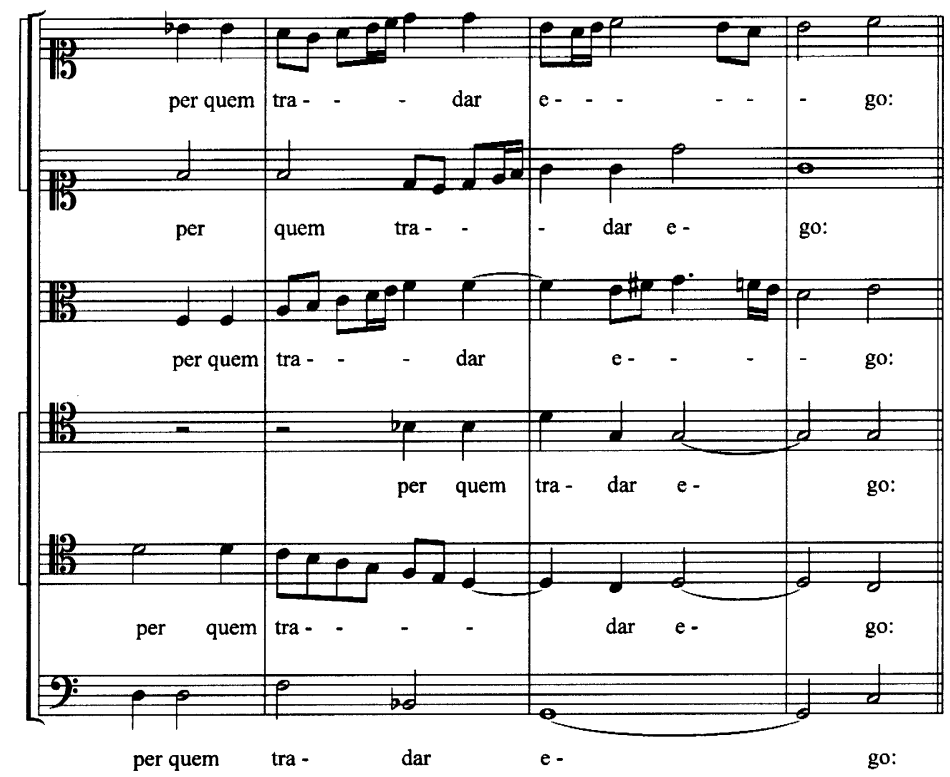

Doch die Diminutionsfiguren der Madrigale und Tastenmusikwerke, als wichtiges Stilmittel schon früh im 16. Jahrhundert in Sätzen von Marc'Antonio Cavazzoni etabliert, sind in Rodios „Ave maris stella" sehr sparsam eingesetzt; offensichtlich und augenscheinlich ist jedes Element virtuoser Spielfreude ausgespart. Abgesehen von ruhigen Durchgangsnoten vermeidet Rodio alles, was einige Praktikertraktate lehren und fantasievoll offerieren: Spielfiguren, Glossen, Verzierungen usw. . Hier darf noch einmal an den Trattado de glosas von Diego Ortiz erinnert werden. Unter der Überschrift „Regla para glosar todos los puntos“19 stellt Ortiz ein Fülle an Verzierungen und Spielfiguren für eine kunstvolle und abwechslungsreiche Instrumentalmusik zusammen. Offensichtlich wollte Rodio hier keine gute Figur machen. So wie er gänzlich auf harmonische Raffinessen und chromatische Experimente verzichtet. Dieses „Ave maris stella“ scheint eine besondere Stellung in dem Repertoire zu haben.

\section{V.}

Der Kirchenhymnus „Ave maris stella“ hat seinen liturgischen Platz in den Marienvespern zwischen dem fünften Psalm und der Versikel. Ein umfangreiches Repertoire an mehrstimmigen Vokalund Orgelkompositionen über diesen Hymnus zeigt, dass dieser zur Agenda der Kirchenkomponisten gehörte; wenn möglich sollte er mehrstimmig musiziert werden. Dabei bediente man sich der Alternatimpraxis, bei der abwechselnd jeweils eine Strophe choraliter und die andere mehrstimmig

19. fol. $20^{\mathrm{v}}-24^{\mathrm{r}}$. Siehe: Nelly van Ree Bernard, Interpretation Of 16th Century Iberian Music On The Clavichord, Buren 1989, S. 68-71. 
ausgeführt wurde. Stand an nicht so bedeutenden Marienfesten während der Vesper kein mehrstimmiger Chor zur Verfügung, so sang man die liturgischen Gesänge im einstimmigen Choral. War zumindest ein Organist anwesend, so konnte dieser die Rolle des mehrstimmigen Chores übernehmen und den einstimmig gesungenen Strophen mit einem mehr oder weniger umfangreichen Orgelvers (Versette) antworten. Für diesen Zweck ist ein beachtliches Repertoire überliefert, aus dem einige

\begin{tabular}{|c|c|c|}
\hline Komponist / Autor & Publikation & Komposition \\
\hline Juan Bermudo & $\begin{array}{l}\text { Comiença el libro llamado Declaración de } \\
\text { instrumentos musicales..., Osuna } 1555 .\end{array}$ & ein Verso \\
\hline $\begin{array}{l}\text { Antonio de Cabezón: } \\
1557 \text { ed. Luis } \\
\text { Venegas de } \\
\text { Henestrosa, } \\
1578 \text { ed. Hernando de } \\
\text { Cabezón }\end{array}$ & $\begin{array}{l}\text { Libro de Cifra nueva / para tecla, harpa, y vihuela, } \\
\text { en el qual se enseña brevemente cantar canto llano, } \\
\text { y canto d'organo, y algunos avisos para } \\
\text { contrapunto, Alcalá de Henares } 1557 . \\
\& \\
\text { Obras de Música para Tecla, Arpa y Vihuela,..., } \\
\text { Madrid } 1578 .\end{array}$ & Mehrere Sätze \\
\hline Hernando de Cabezón & Obras de Música ..., Madrid 1578. & $\begin{array}{l}\text { ein dreistimmiger, teilweise } \\
\text { kanonischer Cantus planus- } \\
\text { Satz }\end{array}$ \\
\hline Girolamo Diruta & $\begin{array}{l}\text { Seconda Parte Del Transilvano Dialogo ..., } \\
\text { Venedig } 1609 .\end{array}$ & ein knapper Verso \\
\hline & Secondo libro di diversi capricci ..., Neapel 1609. & Ein umfangreiches Recercar ${ }^{21}$ \\
\hline Manuel Rodriguez & $\begin{array}{l}\text { Flores De Musica Pera O Instrumento De Tecla \& } \\
\text { Harpa..., Lissabon } 1620 .\end{array}$ & Mehrere Tentos \& Versos \\
\hline Jean Titelouze & $\begin{array}{l}\text { Hymnes De L'Eglise pour toucher sur l'orgue, avec } \\
\text { les fugues et recherches sur leur plain-chànt..., } \\
\text { Rouen } 1623 .\end{array}$ & vier Versets \\
\hline Gion Pietro del Buono & $\begin{array}{l}\text { Canoni, oblighi, et sonate in varie maniere sopra } \\
\text { l'ave maris stella a tre ... et otto voci, e le sonate a } \\
\text { quattro, Palermo } 1641 .\end{array}$ & 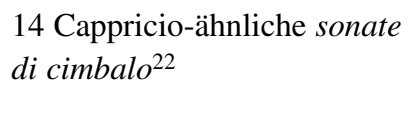 \\
\hline
\end{tabular}

20. Das gleichfalls bedeutende Vokalwerk über „Ave maris stella“ bleibt hier unberücksichtigt.

21. Organum Italicum II, Musica organista dal XV als XVII secolo, Scuole Toscana, Meridionale e Romana, a cura di Andrea Macinanti \& Francesco Tasini, Bergamo 2004. S. 36-38. Edition S. 77-85 (mit Faksimile). [= Macinanti / Tasini]. In Hinblick auf Rodio siehe auch die Rezension in Organ, Journal für die Orgel, 1/06, S. 60ff.

22. Macinanti / Tasini, S. 36. Del Buono stammte aus Neapel, wirkte aber in Palermo. 
repräsentative aber auch einige außergewöhnliche Beispiele aufgeführt werden: ${ }^{20}$

Rodios Fantasie über „Ave maris stella“23 hat bestimmte Merkmale mit den aufgeführten Orgelversen gemeinsam. So finden wir bei de Cabezón, Rodriguez Coelho und Titelouze ausgedehnte Cantus planus-Versetten. Außerdem pflegten die drei Orgelkomponisten besonders die imitatorisch gearbeiteten Versetten im Ricercarestil („Choral-Fuge“), wobei Titelouze der Meister der Synthese beider Formen genannt werden kann. Rodriguez Coelhos Stücke und die von Titelouze begegnen uns außerdem in Form von liturgischen Apparaten: Die Komponisten stellen die vollständige Anzahl an Versetten für die alternatime Ausführung zur Verfügung. Ihre imitatorische Arbeit ist konsequent und dennoch abwechslungsreich. Spielfiguren und virtuose Elemente sind - besonders bei Titelouze immer wieder festzustellen. Bermudos und Dirutas Sätze sind singuläre knappe kontrapunktische Versetten. Als Lehrmaterial veranschaulichen sie, wie ein Cantus firmus-gebundener Orgelvers aussehen kann. Die Primärintention des Anschauungs- bzw. Lehrmaterials teilt Rodio mit Bermudo und Diruta. Mayones Recercar ist von ähnlicher anspruchsvoller kontrapunktischer Faktur wie Rodios Fantasia. Wie dieser publiziert Mayone sein Secondo libro (1609) in Partitur, zwei Systeme pro Seite. Hernando de Cabezóns Ave maristela..., el baxo $C$ ist wie Rodios Fantasia eine strenge Cantus planus-Komposition.

Auch der Titel und Inhalt des Libro de Cifra nueva / para tecla, harpa, y vihuela, en el qual se enseña brevemente cantar canto llano, y canto d'organo, y algunòs avisos para contrapunto weist die Sammlung des Luis Venegas de Henestrosa u.a. als kontrapunktisches Lehrmaterial aus ${ }^{24}$. Doch während dieser und Bermudo in ihren Musikbüchern Bespiele knapper (liturgischer) Sätze geben, beschäftigt sich Rodio erst in seinen Regole di musica mit der Kunst der kontrapunktischen Versi ${ }^{25}$.

Die Melodie des Hymnus „Ave maris stella“ ist mit seinen prägnanten Intervallen eine dankbare melodische Vorlage für mehrstimmige Lehrbuchexempel im 1.Ton. Zunächst einmal ist es Rodio selbst, der in seinen Regole di musica... tutti i canoni sopra il canto fermo (1600) die Kunst des Kontrapunktes u.a. anhand des Marienhymnus lehrt: contrappunti sopra l'Ave Maris Stella ${ }^{26}$. Möglicherweise ließ sich Gioan Pietro Del Buono von Rodios Traktat inspirieren, als er 1641 in Palermo seine Canoni, oblighi, et sonate in varie maniere sopra l'ave maris stella veröffentlichte. Eine ähnlich gewichtige Kontrapunktlehre wie die Rodios erschien in Rom, verfasst von Francesco Soriano: Canoni et oblighi di 110 sorte, sopra l'Ave maris stella $(1610)^{27}$. In diesem Zusammenhang ist unbedingt Pier Francesco Valentini zu nennen, der ebenfalls in Rom die Kunst des Kanons zu seiner Privatwissenschaft entwickelte, ja vielleicht zu einer tiefen Leidenschaft steigerte: 1629 wurde sein Canone ... con le sue resolutioni in più di duemillia modi, libro primo gedruckt. Als melodische Vorlage

23. Neben Rodio und Mayone gibt es noch von dem Neapolitaner Trabaci eine Komposition über den Hymnus. Siehe auch Nicholas Thistlethwaite, The Cambridge Companion to the Organ, Cambridge (UK) 1998, S. 158ff.

24. Siehe Faksimile in: La Música en la Corte de Carlos V. Con la transcripción del "Libro de Cifra Neuva para tecla, harpa y vihuela" de Luys Venegas De Henestrosa, hrsg. von Higinio Anglés, Barcelona 1944, S. 19. [= Libro de Cifra Neuva]

25. Rodio erklärt die Modi mit kurzen vierstimmigen Versi. Cannizzaro, Vol. I., S. 10ff.

26. Cannizzaro, Vol I., S. 11.

27. Paolo Emilio Carapezza, Del Buono, Gioanpietro [Giovanni Pietro], (art.) in: NGroveD, Vol. 5, S. 334. S. Philip Kniseley, Soriano [Suriano, Suriani, Surianus], Franceso, (art.) in: NGroveD Vol. 17, S. 538. 
wählte er nicht den Hymnus „Ave maris stella“, sondern die Antiphon „Salve Regina“, die ja auch Rodio zu einer Fantasia inspirierte. Valentini bot über 2000 Möglichkeiten [= in più di duemillia modi] des Salve Regina-Kanons an ${ }^{28}$.

Paolo Emilio Carapezza vermutet, dass Del Buonos Kanonkollektion von den römischen Canoni Sorianos inspiriert wurde. Und hier kommt eine weitere Persönlichkeit ins Spiel: Der Spanier Sebastián Raval. Dieser wirkte als Kapellmeister des spanischen Vizekönigs in Palermo. 1596 publizierte er dort $I l$ Primo Libro Di Ricercari A Quattro Voci Cantabili Per Liuti, Cimbali, \& Viole d'arco, quattro ò sei opere con parole spirituali in Canoni ad Echo, ad otto, \& à dodici voci, che cantano in quattro parte coniunti, \& divisi i chori. Wenn auch seine vier Kanons nicht den Hymnus „Ave maris stella“ thematisieren ${ }^{29}$, so sind sie dennoch bemerkenswert. Raval gibt diese kontrapunktisch komplexen Sätze einer Publikation bei, die mit den Ricercari schon komplexe polyphone Sätze enthält. Mit den Kanons geht er noch einen Schritt weiter und stellt sich der Öffentlichkeit als Kenner und Könner des strengen Kontrapunktes vor.

Denkbar ist folgender Hintergrund: Soriano und Raval kannten sich persönlich ${ }^{30}$. 1593 waren Soriano und Giovanni Maria Nanino Teilnehmer eines Kompositionswettbewerbes in Rom, den Raval ehrgeizig als musikalischen Streit vom Zaune gebrochen hatte. Es wird berichtet, dass Soriano schon die Kompositionsaufgabe gelöst hatte, als Raval noch über der ersten Zeile brütete. Die römische Blamage mag Raval bewogen haben, in seinem Libro di Ricercari als gelehrter Kontrapunktiker und Verfasser von komplexen polyphonen Instrumentalstücken und Kanons das letzte Wort zu haben. Der Kampf um seinen guten Ruf als Meister des Kontrapunktes ließ ihm keine Ruhe. Als er sich einige Jahre später als Kapellmeister der königlichen Kapelle in Palermo etabliert hatte, provozierte er 1600 einen weiteren musikalischen Wettstreit. Als Kontrahent hatte er sich den jungen Achille Falcone gewählt. Mit List und Tücke konnte er seinen jungen Konkurrenten besiegen. Kurz nach diesem Ereignis starb Falcone. Posthum stellte Antonio Falcone, der Vater des jung verstorbenen Komponisten und Intrigenopfers, mit der Herausgabe von dessen Madrigalen und einem Bericht über den Wettstreit - Alli signori musici di Roma: madrigali a cinque voci ... con alcune opere fatte all'improviso a competenza con Sebastiano Ravalle ... con una narratine come veramente il fatto seguisse, Venedig 1603 - der musikalischen Öffentlichkeit seinen Sohn Achille als kontrapunktischen Meister und als den wahren Gewinner des korrumpierten Wettbewerbs von 1600 vor.

1604 starb dann Raval. Er sollte nicht das letzte Wort behalten. Mit pietätvollem zeitlichem Abstand zeigte Francesco Soriano der Öffentlichkeit - und wenn man von seiner Bedeutung als einer der führenden römischen Komponisten ausgeht - 1610 der Weltöffentlichkeit, was kontrapunktische Kunst sein kann. Offensichtlich war es im späten 16. und frühen 17. Jahrhundert für die Reputation eines

28. Sergio Martinotti \& Agostino Ziino, Valentini [Valentino], Pier [Pietro] Francesco [Pierfrancesco], (art.) in: NGroveD, Vol. 19, S. 495/96.

29. Canones cum octo \& duodecim vocibus. In quatuor partibus. 1. Congregate sunt gentes octo vocum. In quatuor partibus.(Canon cum octo vocibus in quatuor partibus, et divisis quatuor ad unisonum ad Ecco). 2. Haec dies, duodecim vocibus. In quatuor partibus. (Canon duodecim vocibus simul et separatis tribus Choris ad Ecco). 3. Da pacem Domine, octo vocibus. In quatuor partibus. Canon ad Ecco octo vocum in quatuor partibus coniunctis, et separatis). 4. Misit Dominus, duodecim vocibus. In quatuor partibus. (canon Duodecim vocibus in quatuor partibus coniunctis et separatis). Vergleiche Cannizzaro, Vol I., S. 39.

30. Ottavio Tiby, Sebastian Raval - A 16th Century Spanish Musician in Italy, in: Musica Disciplina, Vol. II Fasc. 3 \& 4 (1948), S. 217 - 23. Paolo Emilio Carapezza, Falcone, Archille (art.), in: NGroveD Vol. 6, S. 367/68. Kniseley Art. in NGroveD, siehe Fußnote 25. 
Komponisten nicht unwichtig, als gelehrter und kreativer Kontrapunktiker in der Öffentlichkeit zu glänzen.

Mit den Ricercari a quattro voci und den opere con parole spirituali in Canoni outete sich Raval als souveräner Kontrapunktiker. Und da er durch den misslichen musikalischen Wettstreit seine gute Reputation in Rom eingebüßt hatte, publizierte er seine Ricercari in Palermo. Seine Musik ist geistreich und anspruchsvoll komponiert ${ }^{31}$. Auch verstand er es meisterlich, eine Synthese aus neuer Einfachheit der wortgebundenen Sakralmusik, polyphoner komplexer Satzabschnitte und musiksemantischer Arbeit zu finden, indem er den madrigalen note nere Eingang in seine sakrale Musik verschaffte. Besonders eindrucksvoll zeigt sich dies in seinen Lamentationes Hieremiae Prophetae, die 1594 in fünf Stimmbüchern, also ein Jahr nach dem musikalischen Wettstreit, im Druck erschienen. In
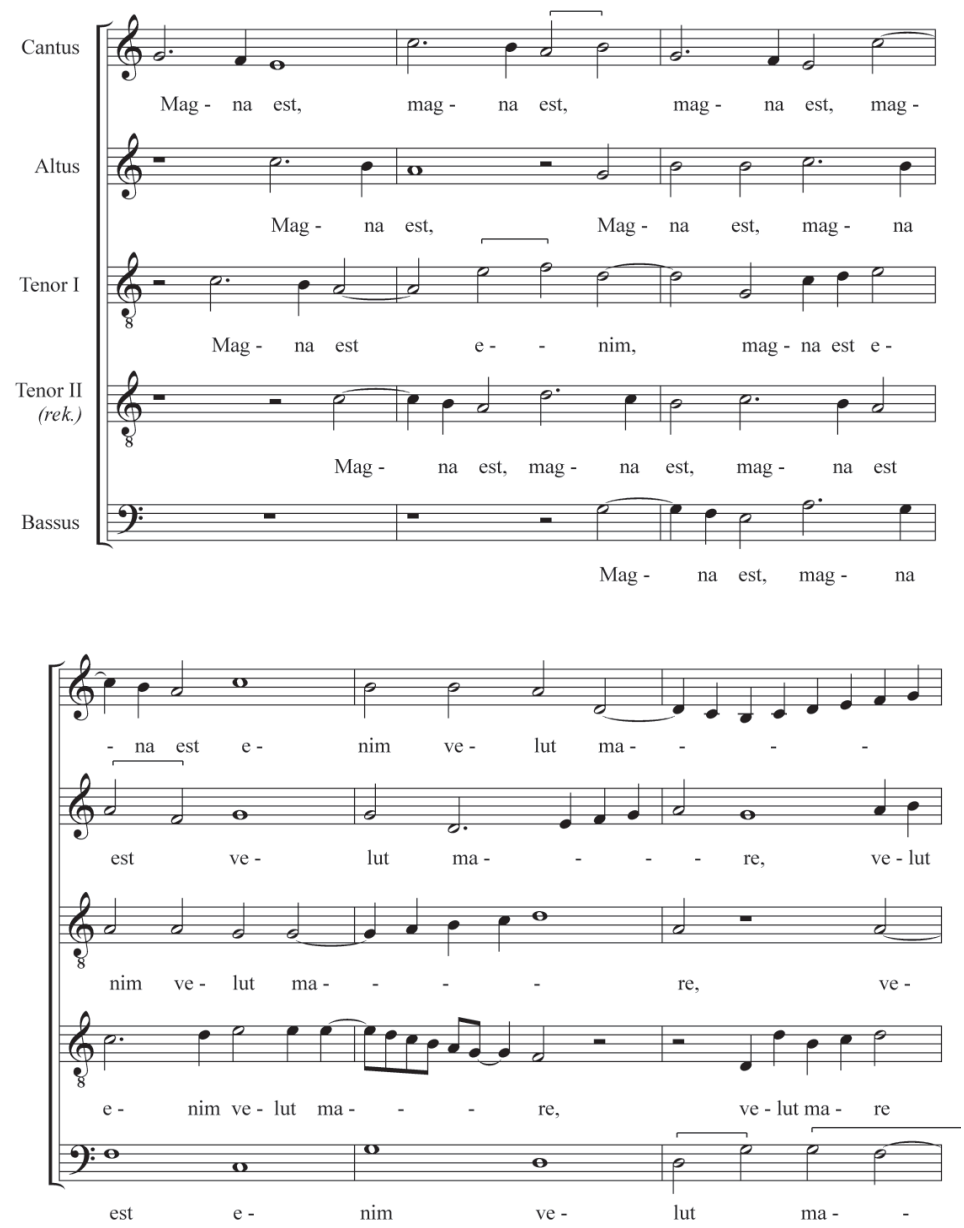

31. Ediert in: Cannizzaro, Vol. II.

32. Johannes Ring, Studien zu den mehrstimmigen Lamentationen im 16. Jahrhundert: Escribano, de Morales und Raval. Hamburg 2001. S. 164/65. 


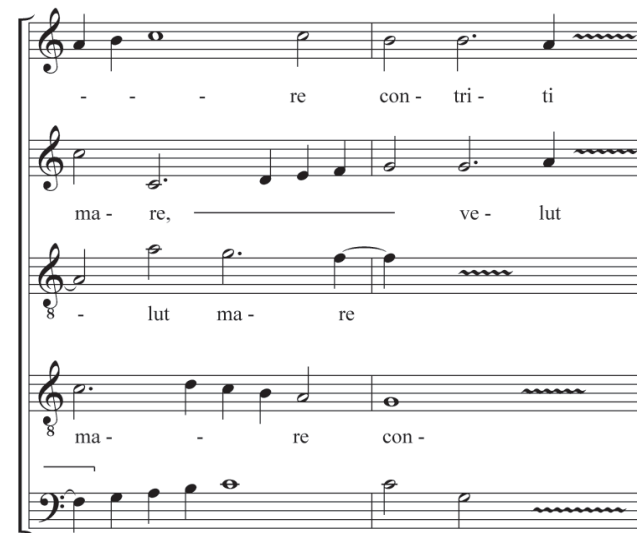

re

einer der Lektionen illustriert Raval mit melismatischen wellenförmigen Tonfolgen die Meereswellen. Diese kannte Raval sicher von seinen Reisen von Spanien nach Italien und von Rom nach Palermo ${ }^{32}$.

In Ravals Lamentationen von 1594 finden wir alle kompositorischen Möglichkeiten mehrstimmiger Satzkunst - freie Polyphonie, imitativen Kontrapunkt, Scheinpolyphonie, Homophonie, Falsobordone und Falsobordonetechnik - Raval zeigt sich als qualifizierter Komponist. Seine Ricercari
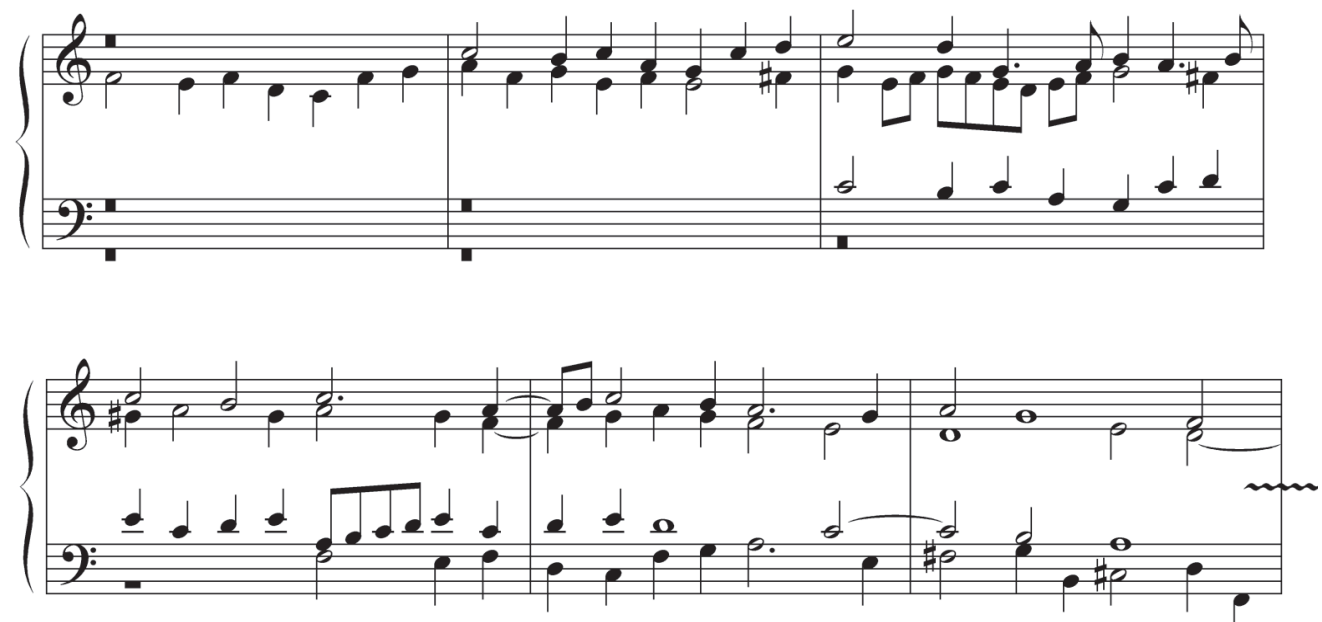

und Canoni (1596) sind gleichfalls Dokumente souveräner Satzkunst und künstlerischer Inspiration, wie der Anfang des Ricercare Sesto Tono zeigt: ${ }^{33}$

33. Siehe Cannizzaro, Vol. II, S. 4 - 124. 
Angesichts der Qualität der Ricercari und der Lamentationes verwundert seine Niederlage beim Wettstreit in Rom sowie seine Intrige gegen den jungen Falcone in Palermo etwas.

Nach diesen Ereignissen können wir uns wieder Rocco Rodio zuwenden, der zunächst noch wie ein Zaungast wirkt. Aber während man sich in Palermo um die wahre Kunst des Kontrapunkts stritt, veröffentlichte Rodio seinen Traktat mit den contrappunti sopra l'Ave Maris Stella. Sein Traktat geht den römischen Kanonwerken voraus. Rodio und Soriano gehören verschiedenen Generationen an; persönlich kannten sie sich wohl nicht. Vermutlich hatte sich der aus Bari stammende Rodio vor seiner Übersiedelung in die Hauptstadt Kampaniens eine Zeitlang in Rom aufgehalten. Dort erschien 1562 sein Buch mit Messen zu vier bis sechs Stimmen Missarum decem liber primus. Es ist gut vorstellbar, dass Rodio den Kontakt mit Rom weiter pflegte und mit dem musikalischen Leben Roms weitgehend vertraut war.

Ein weiterer wichtiger Faktor im Musikleben Neapels war die schon oben erwähnte Existenz des Oratorio dei S Filippo. Ab 1586 nahmen römische Musiker über diese Bruderschaft deutlichen Einfluss auf das Musikleben in Neapel. Man darf das Oratorium getrost als Anlaufstelle, als erste Adresse römischer Musiker bzw. ihrer kompositorischen Ideen und Arbeiten in Neapel werten, wodurch die stark iberisch und flämisch geprägte Musikszene der Stadt allmählich ein zusätzliches gewichtiges römischen Ingredienz erhielt. Sicher war die Musikschlacht zwischen Raval und den beiden führenden römischen Komponisten dort ein Gesprächsthema. Und wahrscheinlich galten römische Komponisten von dem Kaliber eines Sorianos und Naninos als Leitbilder für einen professionellen Musiker. Im Werk dieser Komponisten und schon früher, beispielsweise bei Giovanni Pierluigi da Palestrina, hatte sich, als Folge der Verlautbarungen des Konzils von Trient zur mehrstimmigen vokalen Kirchenmusik, ein transparenter und klarer Stil entwickelt, der eine gute Synthese mit dem aktuellen musikalischen Geschmack der Vereinfachung polyphoner Schreibweise einging. Ihren akademischen bzw. kontrapunktischen Leidenschaften mussten die Komponisten nun in instrumentalen bzw. didaktisch ausgerichteten Musikgattungen bzw. Disziplinen frönen. Diese vielschichtigen musikalischen Einflüsse in Neapel und seine römischen Erfahrungen mögen Rodio bewogen haben, seinen eigenen musikalischen bzw. musiktheoretischen Standpunkt auszuarbeiten und publik zu machen.

\section{VI.}

Die Bedeutung, die dem Ricercare und der Fantasie als instrumentalen bzw. tasteninstrumentalen Gattungen zukommen, spricht John Gillespie an: ,... ricercar and fantasia, forms governed by highly developed contrapuntal rules. ... The ricercar is more disciplined than the fantasia and reperesents one of the highest forms of contrapuntal art." ${ }^{34}$ Vornehmlich im Ricercare - in Spanien der tiento und in Portugal tento - und mit dem Kanon demonstrierten die Komponisten im 16. und frühen 17. Jahrhundert die gelehrte, respektive kontrapunktische Satzkunst. Nach den besonders in Venedig gepflegten Ricercari für Laute sind es die Namen Girolamo Cavazzoni, Giaches Buus und Adrian

34. John Gillespie, Five Centuries Of Keyboard Music, New York 1965. S. 30. Am Ende dieser Tradition stehen beispielsweise die Beiträge von 1747 von Johann Sebastian Bach (,Musikalisches Opfer“). 
Willaert, die in den 1540er Jahren maßgebliche Beiträge zum kontrapunktischen Ricercare leisten. Gerade bei Buus wird die Entwicklung vom motettisch konzipierten Satz zum Singen und zum Spielen auf der Orgel und auf anderen Instrumenten [,da cantare et sonare d'organo \& altri stromenti“] in Stimmbüchern gedruckt hin zum tastenmusikalischen Werk deutlich. Buus selbst hat vier seiner ursprünglich in Stimmbüchern publizierten Ricercari für Orgel bzw. Tasteninstrument bearbeitet: Intabolatura d'Organo di Ricercari di M. Giacques Buus, Organista dell illustrissima Signoria di Venetia in San Marco. ..., Venedig 154935. Die kunstvollen und umfangreichen Ricercari von Buus, der in der Zeit ihrer Entstehung als Organist am Markusdom in Venedig wirkte, sind Paradebeispiele komplexer, mitunter gelehrter niederländischer Kontrapunktik. Die vier Orgelintabulierungen integrieren und entfalten maßvoll instrumentale Figuren und Verzierungen.

Rodios Ricercari haben keinen einheitlichen Bauplan. Sie sind konsequent stimmig und imitativ gearbeitet, in ruhigem, ausgeglichenem Kontrapunkt. Dann und wann lässt Rodio tastenspielerischen

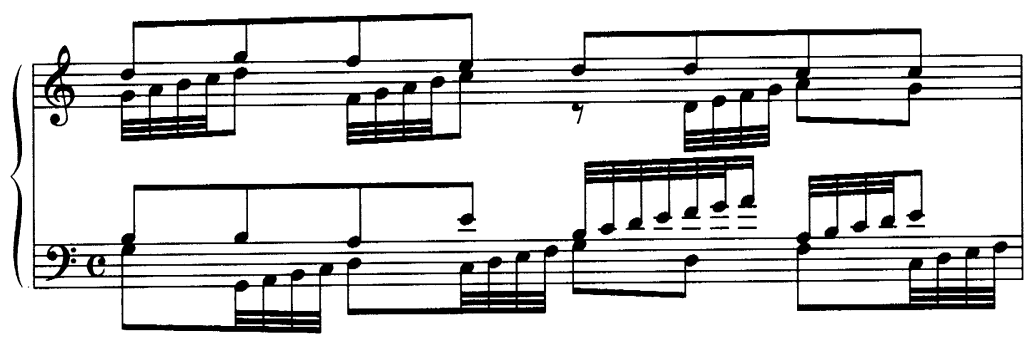

Virtuosität aufscheinen, am eindrucksvollsten am Ende des ersten Ricercare, worauf W. Apel hingewiesen hat $^{36}$.

Eine vorsichtig formulierte gattungsformale Tendenz scheint zu sein, dass Fantasien mitunter kontrapunktisch und strukturell nicht so konsequent gearbeitet sind wie die Ricercari. Doch tatsächlich wurden die Begriffe immer wieder synonym verwendet. Die Quellenlage im Italien des 16. und frühen 17. Jahrhunderts bestätigt die Austauschbarkeit der Begriffe Ricercare und Fantasia. Anschauungsmaterial hierfür liefert Girolamo Frescobaldi. Seine Fantasie a quattro von 1608 (Mailand) sind kontrapunktisch und formal genauso diszipliniert gearbeitet wie beispielsweise die Ricercari von Rodio. Dagegen sind Frescobaldis Ricercari von 1615 (Rom) formal wesentlich abwechslungsreicher aufgebaut als seine frühen Fantasie ${ }^{37}$.

35. Otto Kinkeldey hat die vielfältigen Ausprägungen des Ricercares (Tientos) unter Einbeziehung des Fantasiabegriffs gut beschrieben: Orgel und Klavier in der Musik des 16. Jahrhunderts, Hildesheim u.a. / Wiesbaden 1910/1984, S. 136-47. [= Kinkeldey] Zu Buus' 18 Ricercari, die in drei Drucken erschienen, siehe: H. Colin Slim, Buus, Jacques [Jacob], (art.) in: NGroveD Vol. 3, S. 525/26. Slim schrieb die außerdem die grundlegende Arbeit zum frühen italienischen Ricercare: The Keyboard Ricercar and Fantasia in Italy ca. 1500-1550, Harvard University 1960. 10 Ensemble-Ricercari von J. Buus sind in der portugiesischen Handschrift Biblioteca Geral da Universidade de Coimbra, Ms. 48 von ca. 1560 überliefert.

36. Vergl. Apel1967, S. 179ff.

37. Vergl. Field, S. 381/82. 
Ein wenig Verwirrung stiftet ein weiterer Komponist aus Neapel. Ascanio Mayone versieht seinen Druck von 1603 mit dem launischen Titel Primo libro di diversi capricci per sonare. Das Buch beinhaltet nicht ein Stück mit der Bezeichnung Capriccio, wie wir es von Girolamo Frescobaldi her kennen. Eher ist der Buchtitel als bescheidene Selbstdegradierung des jungen Komponisten zu werten, der seine „gut gelaunten Einfälle“ der Öffentlichkeit übergibt. Wie bei Rodio enthält dieses Buch u.a. vier Ricercari. Sechs Jahre später greift Mayone den Titel in einer weiteren Publikation dieser Art auf: Secondo libro di diversi capricci per sonare. Diesmal sind es fünf Ricercari kombiniert mit Toccaten, Kanzonen und Variationen. Bezeichnend ist Mayones Kommentar im Vorwort, wo er einräumt, dass spielfreudige Passagen auf den ersten Blick gegen das kontrapunktische Lehrwerk verstoßen. Verstörte Musiker vertröstet er mit den Ricercari am Anfang des Buches ${ }^{38}$. Damit steht für Mayone ein bis zwei Generationen nach Rodio das Ricercare bzw. das Beherrschen der kontrapunktische Lehre immer noch an erster Stelle. Bestätigt wird dies in Neapel zeitgleich mit Ricercate: canzone franzese, capricci, canti fermi, gagliarde, partite diverse, toccate, durezze, ligature, consonanze stravaganti: Opera tutte de fonare: a quattro voci: Libro primo und zwölf Jahre später nochmals mit Il secondo libro de ricercate \& altri varij capricci con cento versi sopra li otto finali ecclesiastici..., jeweils von Giovanni Maria Trabaci. Noch zwei Generationen später hält der Franziskaner Fra Giovanni Battista Fasolo die Ricercari für so wichtig, dass er acht Stück für sein Annnuale (Venedig 1645) komponierte. Auch wenn in Fasolos Opera omnia liturgische Orgelverse für Vesper und Messe im Vordergrund stehen, so führt er doch die Ricercari als erste Gruppe der freien Orgelsätze auf ${ }^{39}$.

VII.

Antonio Valentes Buch Intavolatura a cimbalo (1575) enthält eine Fantasia, ein frei komponiertes zweiteiliges Musikstück. Ausdrucksstarke dissonanzreiche Passagen wechseln mit toccatenmäßigen virtuosen Abschnitten ab. Seine Ricercari beginnen mit einer stimmigen Exposition des Soggetto. Im weiteren Verlauf wird der Satz hauptsächlich von Ideen, die sich aus den grifftechnischen Möglichkeiten des Tasteninstrumentes ergeben, und kaum von konsequenter kontrapunktischer Arbeit bestimmt. Valentes Fantasia und die Ricercari unterliegen in ihrer Faktur hauptsächlich spieltechnischen Intentionen.

Der eigentliche Unterschied zwischen Rodios kontrapunktischen Fantasien und Ricercari besteht darin, dass die Fantasien einen Cantus prius factus als Grundlage haben. Die Notenbeispiele der Fantasie „Ave maris stella“ (Siehe III.) veranschaulichen, dass die drei polyphonen Stimmen keinen oder nur einen marginalen Bezug zum Cantus firmus haben. Die Abschnittbildung in Rodios Fantasie

38. ,... quando si sona con passagi, o si adornano opere di passagi, sempre vi passano alcune note false contra la regola del contrapunto, senza le quali è impossibile, che bello effetto faccia; per questo dico a chi questa opera vederà, che non si scandaliza, e mi giudica di poco osservatore delle regole del contrapunto, delle qual sempre ho fatto professione quando se è possibile col mio rozzo ingegno di osservare, e che di questo vuol star sicuro, e li dispiacesse questo modo di sonare; potrà vedere, e servirse delle Recercate poste nel principio del presente, (como ho fatto anco al Primo Libro de miei Capricci)...". Siehe: Ascanio Mayone, Diversi Capricci Per Sonare Libro I Napoli 1603, hrsg. von Christopher Stembridge, Padua 1981. S. III. [= Mayone] S. II \& III.

39. Vergleiche Vorwort zu: Giovanni Battista Fasolo, Annuale "Venedig 1645”, hrsg. von Rudolf Walter, Heidelberg 1977, 
richtet sich nach der melodischen Vorlage. Entsprechend der Melodie- bzw. Textgestalt der Melodie respektive Cantus firmus treten Imitationspunkte auf. Entsprechend der vier Melodieabschnitte des Hymnus „Ave maris stella“ stellt Rodio vier Imitationspunkte vor. Diese Imitationspunkte haben nicht das Gewicht von Expositionen der Soggetti in den Ricercari. Rodios Fantasie sind kontrapunktisch anspruchsvoll und komplex gearbeitet. Sie demonstrieren den Tastenmusikern, wie man einen kunstvollen und geistreichen Satz über einen Cantus firmus gestaltet. Die vier Fantasie sind eine gute Ergänzung der fünf Ricercari, die gleichfalls eine Kombination aus inspirierter Musikalität und meisterlicher Kontrapunktik sind. Rodios ganz persönliche Intention und Interessenlage, die 1600 nochmals in seinem Praktikertraktat Regole di musica bestätigt wird, galt der kontrapunktischen Satzkunst, die er anhand seiner Fantasie und Ricercari lebte und dokumentierte.

Das bunte Nebeneinander von distinguierter kontrapunktischer Kunst Rodios und erfrischend unkonventioneller Spielfreude Valentes bescherte Neapel eine kaleidoskopische musikalische Vielfalt mit freien Entfaltungsmöglichkeiten auf einem interessanten musikalischen Markt mit einer funktionierenden Musikpresse.

VIII.

Aus heutiger Sicht scheint es bei Rodios Fantasie Ausführungsprobleme zu geben. Stellenweise
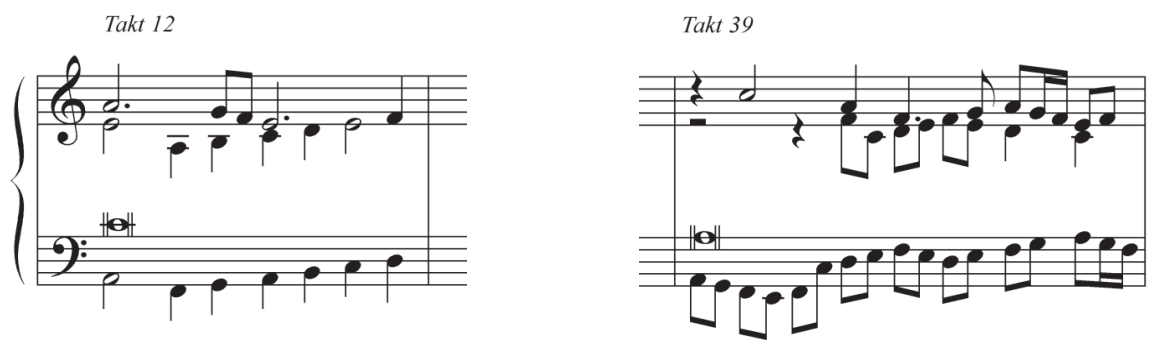

liegen die Stimmen so weit auseinander, dass sie nicht zu greifen sind, selbst wenn das Pedal eine Stimme übernehmen würde. Zwei Beispiele aus der Fantasia sopra Iste Confessor verdeutlichen $\operatorname{dies}^{40}$ :

Dieses Problem hat schon Willi Apel angesprochen. Das Problem löst sich mit dem Hinweis, dass die süditalienischen Orgeln engere Tasten hatten, auf denen sich Dezimen greifen lassen ${ }^{41}$. Außerdem geht Rodios tastenmusikalischer Satz von einer kurzen Oktave im Bass aus, eine typische Einrichtung der Tasteninstrumente, mit der im hohen Maße schon 1555 Juan Bermudo seine Orgelsätze konzipierte. Bei einem mäßigen Grundschlag in Verbindung mit der besagten instrumentalen Situation und dem bedarfsweisen Wechseln der mittleren Stimmen zwischen den Händen sind Rodios Fantasie und

40. Nach Cannizzaro, Vol. II, S. 127 \& 129.

41. Apel1967, S. 120. 
Ricercari gut ausführbar.

Eine weitere Bestätigung dafür, dass man in Neapel von einer kurzen Bassoktave ausgeht, findet sich in Valentes Intavolatura de Cimbalo. Valente benutzt eine der spanischen Orgeltabulatur ver-

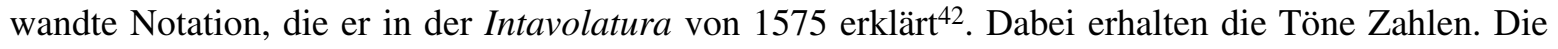
Zuordnung der Zahlen bei Valente bestätigt schließlich die kurze Bassoktave in der Tastenmusik

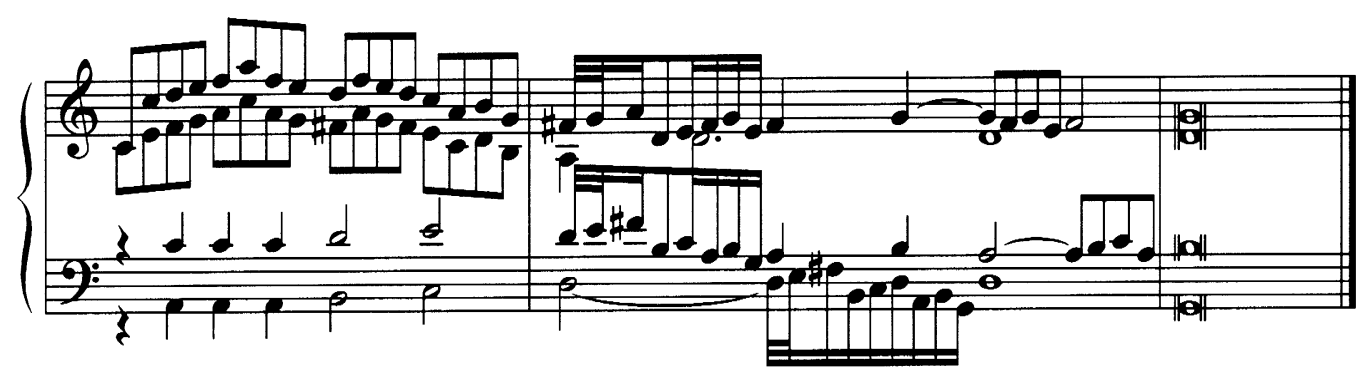

Neapels. Schmalere Tasten erwartet immer wieder A. Mayone, was sich anhand der Schlusspassage der Canzon Francese Terza aus dem Primo Libro di Diversi Capricci (1603) eindrucksvoll dokumentieren lässt: 43

Bei Mayone treten gerne Dezimen auf, besonders in der linken Hand. Engere Tasten und die kurze Bassoktave erlaubt es den Komponisten in Neapel einen im Stimmumfang ausgedehnten Satz zu schreiben, umfangreicher disponiert als beispielsweise Girolamo Frescobaldi in Rom, wo Dezimen eine Ausnahme sind. Den spielerischen Einsatz der kurzen Oktave im Bass und den schmaleren Tasten

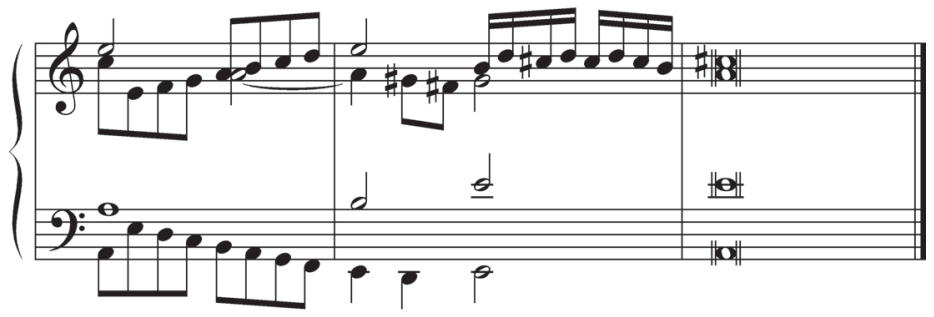

lassen wir uns ein weiteres Mal von Giovanni Maria Trabaci bestätigen, dessen Schlusskadenz der Toccata prima à quattro aus dem Secondo Libro de Ricercate, \& ... (Neapel 1615) einen bemerkenswerten Stimmenambitus aufweist ${ }^{44}$.

42. Antonio Valente, Ricercars, Chansons and Dances from Intavolatura de Cimbalo 1576, hrsg. von Bernard Thomas, London 1981. S. I.

43. Nach Mayone, S. 21.

44. Faber Early Organ Series, European Organ Music Of The Sixteenth \& Seventeenth Century, Vol. 17, Italy c.1600-1635. E. Pasquini - De Macque · Mayone · Trabaci · Cima · Diruta - Banchieri · Frescobaldi, London 1988, S. 15. 
Über die tastensinstrumentale Situation bzw. die praktische Ausführbarkeit großer Griffe auf dem Tasteninstrument brauchte sich der Kapellmeister der spanischen Hofkapelle in Palermo nur wenig Gedanken zu machen. Ravals Il Primo Libro Di Ricercari A Quattro Voci ist für Liuti, Cimbali, \& Viole d'arco disponiert. Ein kleines Ensemble soll den vierstimmige Satz ausführen. Raval sieht sich in der Tradition des italienischen Ensemblericercares stehen. Im Vorwort erwähnt er zwei seiner berühmtesten norditalienischen Komponistenkollegen “... il grand' Adriano Villaert, \& Cipriano Rore suo discepolo grandissimi contrappuntisti...", die sich als Komponisten u.a. von Ensemblericercari - imitativ gearbeitete Ricercari im Motettenstil - einen Namen gemacht haben (VI. $)^{45}$.

Offenbleiben muss, ob Rodio, Valente, Mayone und die anderen Organisten Neapels das Pedal einsetzten. Ihre Clavierbücher enthalten keinen entsprechenden Hinweis. Einige italienische Orgeln dieser Zeit verfügten über ein angehängtes Pedal. Und die Quellen der Orgelmusik - beispielsweise kann Frescobaldis Toccata sesta per l'Organo "sopra i pedali e senza” im 2. Toccatenbuch von 1627 genannt werden - sprechen das Pedalspiel $a^{46}{ }^{4}$. Womöglich hat Rodios Fantasie sopra „Ave maris stella“ Bezug zur früheren Tenormotette, den Tenor-Cantus firmus allerdings zeitgemäß in einen geschmeidigen polyphonen Satz mit knappen Imitationspunkten gegossen. Vergleichbares finden wir
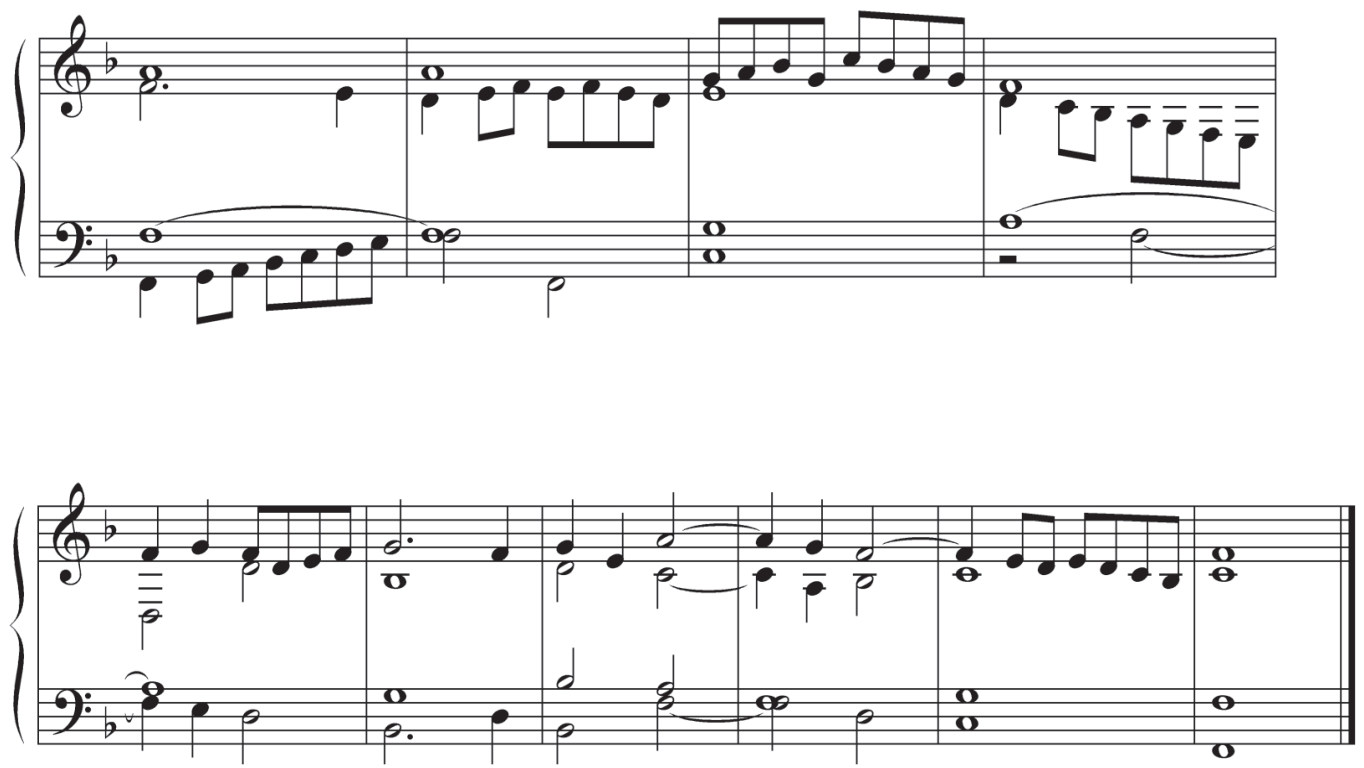

45. Vergl. Cannizzaro, Vol. I, S. 33 \& 35, Caldwell, S. 836 und Reese, S. 549. Inwieweit Raval und die hier genannten Komponisten aus Neapel von den Cantus firmus-gebundenen Stücken aus dem 2. Buch des Praktikertraktats, Trattado de glosas sobre clausulas..., von Diego Ortiz (1553) beeinflusst sind, wäre eine weitere Untersuchung wert.

46. Siehe auch Klotz, S. 137.

47. Nach Libro de Cifra Neuva, S. 120. 
auf der iberischen Halbinsel in der Zeit vor und nach Rodio: Antonio de Cabezóns berühmter Orgelsatz über den vierstimmigen Hymnus „Pange lingua“ von Juan Urreda (= Johannes Vrede) im Libro de Cifra nueva (1557): ${ }^{47}$

De Cabezón bearbeitet eine Tenormotette über den Hymnus „Pange lingua“. Andere Sätze de Cabezóns über diese Hymnusmelodie ${ }^{48}$ und über „Ave maris stella“ (vier- oder dreistimmig) sind ähnlich gearbeitet. Zwei Generationen nach Rodio publiziert Rodriguez Coelho 1620 Hymnussätze, die den Cantus firmus als Cantus planus jeweils einer Stimme zuordnen. Im Gegensatz zu anderen Sätzen mit liturgischer Bindung bezeichnet der Portugiese folgende Hymnussätze nicht als verso (= Vers, Versette): Primeira Ave maris stella sobre o canto chão do tiple em minimas. Segunda Ave maris stella sobre o Canto chão do Contralto. Terceira Ave maris stella sobre o Canto chão do Tenor. Quarta Ave maris stella sobre o Canto chão do Contrabaxo. Diese Sätze über "Ave maris stella" und "Pange lingua” zählt Rodriguez Coelho direkt nach den Intabulierungen der Chanson „Susane un jour“ von Orlando di Lasso ${ }^{49}$. Diese ordnet er in der Vorrede noch den Tentos zu. Damit definiert er nicht die musikalischen Gattungen Tento und Verso, sondern unterscheidet ihre Intention. Die Cantus planusSätze über die Hymnusmelodien lehren die Kunst, wie man einen Cantus firmus als Cantus planus
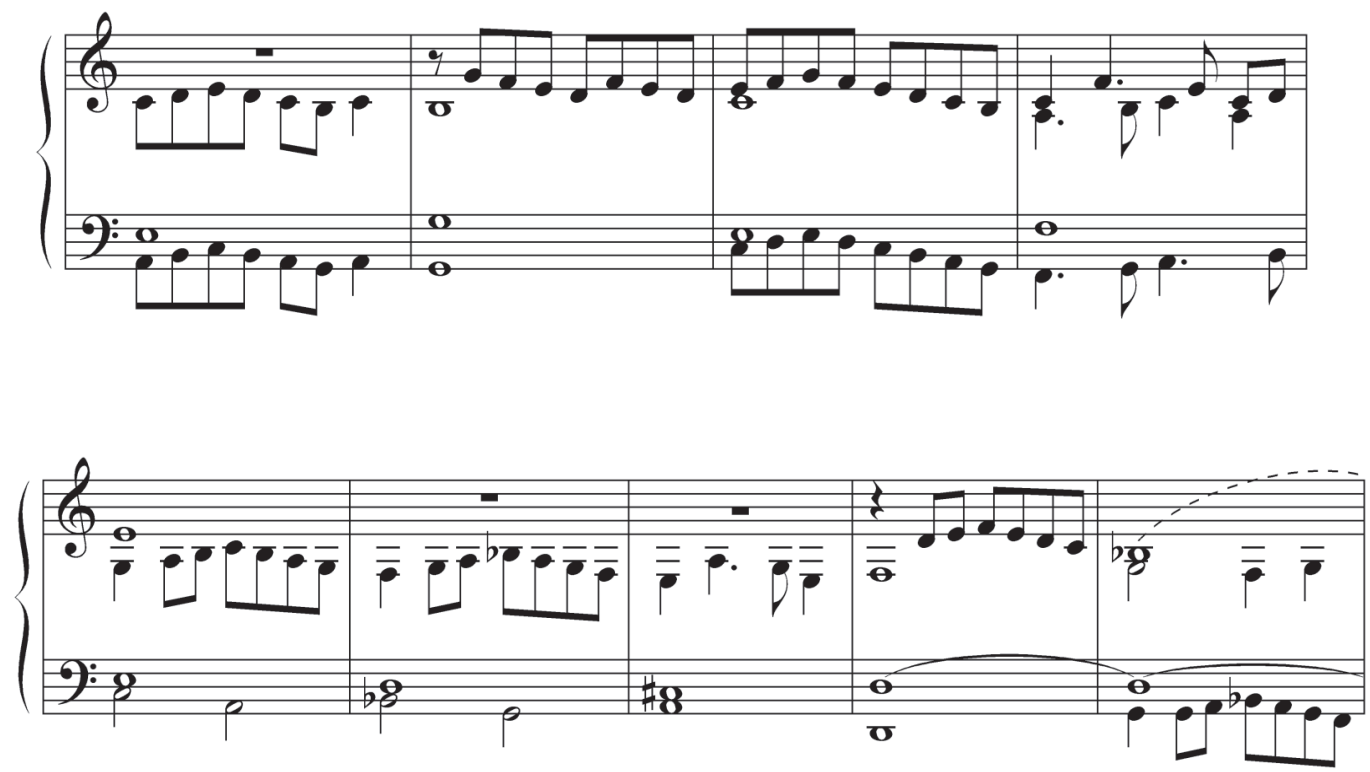

48. Spanische Melodie „more hispano“ des Hymnus „Pange lingua“. U.a. auch bei Bermudo und Rodriguez Coelho.

49. ,..., com mais quatro Susanas... Tentos (que asi se podem chamar) sobre o canto chão da Susana, ... Mais quatro Pangelinguas... Quatro Ave Maris stellas... ." Die anschließenden Sätze über “Ave maris stella” bezeichnet Rodriguez Coelho als Verso. Vergl. Manuel Rodrigues Coelho, Flores De Musica Pera O Instrumento De Tecla \& Harpa, Vol. I hrsg. von Macário Santiago Kastner, Lissabon 1976. S. XL. Edition in: Manuel Rodrigues Coelho, Flores De Musica Pera O Instrumento De Tecla \& Harpa, Vol. II hrsg. von M. S. Kastner, Lissabon 1961. S. 33. 


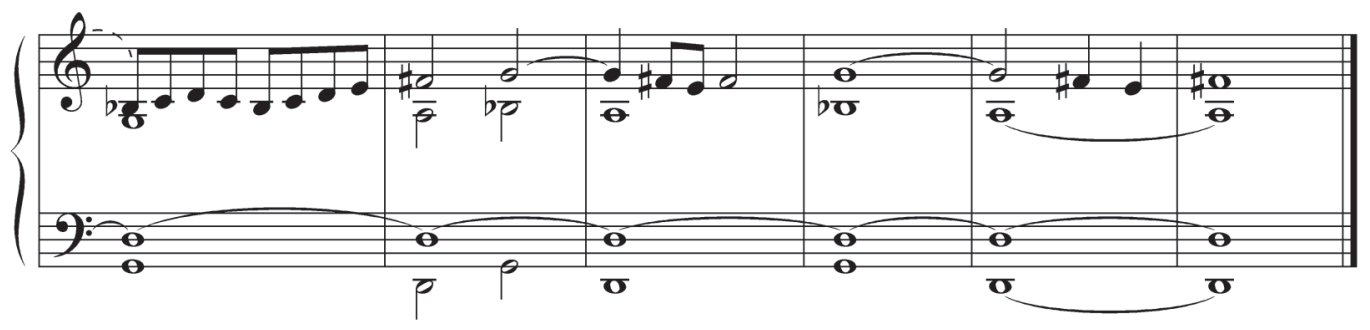

jeweils in einer anderen Stimme durchführt und dabei die anderen Stimmen polyphon setzt. Die Endpassage aus dem 3. Satz über „Ave maris stella“ im Tenor zeigt die Kunst des königlichen Organisten aus Lissabon:

Was wir bei Rodio beobachteten, trifft auch bei Rodriguez Coelho und de Cabezón zu: Die Zeilenbildung des Hymnustextes diktiert die Satzstruktur. Die polyphonen Stimmen imitieren sich mehr oder weniger deutlich. Die iberischen Komponisten schreiben manchmal Vorimitationen, auf die Rodio verzichtet. Wie Rodriguez Coelho definiert Rodio nicht die formalen Gattungen Fantasia und Ricercare. Das Verwenden von zwei Begriffen innerhalb eines Druckes, die für polyphone bzw. imitatorisch gearbeitete Musik stehen, begründet sich nicht in einem Definitionsversuch, sondern in der Differenzierung. Rodios Auffassung des Ricercares steht für das Erforschen imitativer Satzmöglichkeiten frei erdachter musikalischer Themen, während die Fantasia für polyphone Satzkunst über einen Cantus planus steht. Vergegenwärtigen wir uns an dieser Stelle, dass es eine der vornehmen Aufgaben bzw. Künste der Organisten war und ist, über den Choral zu improvisieren, d.h. einen stimmig konzipierten polyphonen Satz über einen Cantus firmus zu kreieren.

\section{IX.}

Die langen Cantus firmus-Töne in Rodios Fantasien laden dazu ein, sie pedaliter auszuführen. Allerdings hatten die meisten italienischen Orgeln des 16. Jahrhunderts kein eigenständiges Pedal, bzw. war das Pedal klanglich nicht für den prägnanten Vortrag von Melodien eingerichtet. Somit fällt die klangliche Wiedergabe Rodios Fantasia sopra „Ave maris stella“ anders aus als beispielsweise „Ave maris stella“ - Versets von Jean Titelouze, die als repräsentatives Beispiel (siehe V.) angeführt wurden. Titelouze sieht den Einsatz von zwei eigenständigen Manualen für die Hände und ein umfangreiches Pedalwerk in Achtfußlage (8́) vor ${ }^{50}$. Jeweils die erste (1 ${ }^{\text {er }}$ Verset) einer Versettenfolge über 12 Kirchenhymnen ist eine Cantus firmus- bzw. Cantus planus-Versette. Die Hymnusmelodie liegt jeweils

50. ,... les faisant construire en plusieurs lieux de la France avec deux claviers separés pour les mains, \& un clavier de pedales a l'unisson des jeux de huict pieds, contenant vingt-huict ou trente tant feintes que marches...", in: Jean Titelouze, Euvres complètes d'Orgue, hrsg. von Alexander Guilmant, Mainz u.a. 1967. S. 9. [= Titelouze]. Notenbeispiel nach Titelouze, S. 44. In den jeweiligen zwei bzw. drei Folgeversetten gewinnt Titelouze seine fugues \& contre-point gerne durch Paraphrasen der Hymnusmelodieabschnitten. Die Zitatauszüge sind dem Vorwort Au Lecteur (1623) in Titelouze, S. 9-11 entnommen. 


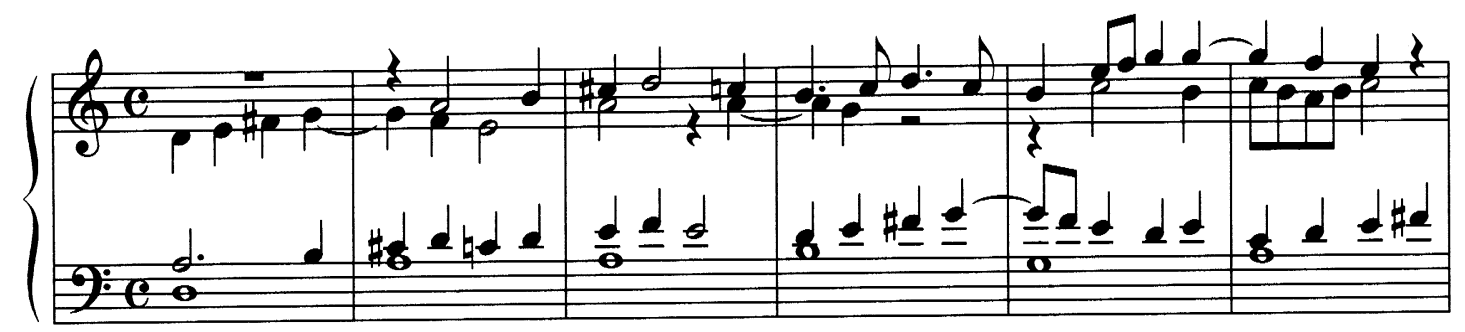

in der unteren Stimme. Die Lage der Hymnusmelodie ist überwiegend gut singbar und lässt sich in der Alternatimpraxis problemlos vom Vokalchor aufgreifen.

51. Italy c.1517-1599, S. IX/X und Klotz, S. 132ff.

52. Klotz, S. 137/38. Auf einer modernen Orgel lässt sich Rodios Fantasia über „Ave maris stella“ ausführen, indem das an das Manual gekoppelte Pedal die Bassstimme übernimmt. 
Wie Rodio setzt Titelouze sofort mit der Cantus firmus ein. Die drei kontrapunktischen Stimmen beziehen sich in kleinen Imitationsfeldern immer wieder aufeinander. Die Installation dieser Imitationsfelder orientiert sich an der textgebundenen Struktur der Hymnusmelodie, um einige Übereinstimmungen zwischen Fantasia auf der einen und Verset auf der anderen Seite zu nennen. Doch beziehen sich bei Titelouze die drei kontrapunktischen Stimmen der Eröffnungsversets fast immer auf die Hymnusmelodie [, ... me contraint dỷ conformer les fugues \& contre-point.“]; offensichtlich das Konzept seiner Arbeit. Beide - Rodio und Titelouze - sind zu Recht als führende Kontrapunktiker ihrer Zeit zu bezeichnen. Sie sehen das Tasteninstrument als geeignetes Mittel, um den Kontrapunkt darzustellen bzw. zu lehren [, ... De sorte quón peut toucher sur lÓrgue du contre-point meilleur qu'estant chanté, \& d'autre aussi au contraire."]. Mit dieser Erkenntnis knüpfen sie bewusst oder unbewusst bei Juan Bermudo an.

Gegen diese klangliche, den Cantus firmus exponierende Option und die Entwicklungen im französischen Orgelbau [,,en plusieurs lieux de la France“] ab dem späten 16. Jahrhundert steht in Italien eine andere Orgelbautradition und Klangästhetik, die sich natürlich auch in der Spielweise und letztlich in der Satzweise niederschlägt. Die meisten italienischen Orgeln zwischen dem 15. und 17. Jahrhundert hatten nur eine Tastatur ${ }^{51}$. Einige Instrumente hatten ein Pedal mit einem Tonumfang von meist nicht mehr als einer Oktave. Wo das Pedal umfangreicher eingerichtet war, hatten die Organisten die Freiheit, je nach Qualität und Quantität der Bassstimme oder des Cantus firmus, diese oder jenen pedaliter auszuführen ${ }^{52}$. Da uns die genauen Dispositionen der Tasteninstrumente Rodios nicht bekannt sind, schließen wir mit den soweit bekannten Tatsachen und den sich daraus ergebenden Schlussfolgerungen: Auf der klassischen italienischen Orgel (in Neapel) mit den schmaleren Tasten kombiniert mit der kurzen Bassoktave verliert Rodios Fantasia über „Ave maris stella“ ihre ursprünglich befürchtete Sperrigkeit. Nur auf einem solchen Instrument sind die Dezimen (bis hin zu Duodezimen) als Griff in einer Hand praktizierbar. Rodios Satz ist somit flüssig spielbar und entfaltet eine erfreuliche Klangwirkung. Eine durchsichtig klingende, organisch gearbeitete Polyphonie mit ausgezirkelter Stimmführung fließt über den beständigen Tönen des Cantus firmus im Tenor.

\section{Ricerca ultima}

Titelouze's Vorwort “Au Lecteur” ist ein pointiert verfasster Praktikertraktat, der die Philosophie der Musik kurz anspricht, prominente Musiktheoretiker als Gewährsleute anführt (z.B. Rodios Zeitgenossen Gioseffo Zarlino und Francisco de Salinas ${ }^{53}$ ), auf Kontrapunkt, Formenlehre und Aufführungspraxis eingeht, die instrumentale Situation andeutet und schließlich konkrete pädagogische Vorschläge formuliert. Was Titelouze 1623 in einem Band der Öffentlichkeit vorlegt, verteilt sich bei Rodio auf zwei Musikbücher (1575 \& 1600). In diesem Kontext mit dem etablierten Komponisten

53. Inwieweit F. de Salinas das Musikleben Neapels beeinflusste, bleibt noch zu klären. Siehe Cannizzaro, Vol. I, S. 3.

54. Rodios "Ave maris stella" u.a. als MP3 unter http://keyboardmusic.tk.

55. Siehe Antologia De Organistas Do Século XVI, transkripiert von Cremilde Rosado Fernandes, Vorwort von M. A. Kastner, PM XIX, Lissabon 1969, bes. S. 78-82. 
de Cabezón, und später mit Rodriguez Coelho und Titelouze steht Rodio mit seiner Hymnuskomposition "Ave maris stella". Sie wollten die jeweils aktuelle polyphone Tastenspielkunst fantasievoll und kontrapunktisch durchdacht über Cantus planus-Tönen, gerne auch, wie in der Tenormotette in der entsprechenden Stimme des (vierstimmigen) Orgelsatzes, entfalten.

Natürlich wusste Rodio um die spieltechnischen Möglichkeiten der Musik für Tasteninstrumente, von den lebendigen und virtuosen Spielfiguren und Griffmöglichkeiten mit spannenden harmonischen Wendungen. Rodio vereinigt beide Polaritäten der Tastenmusik, die der polyphon komplex durchdachten Anlage mit jener der virtuosen Spielfreude. Doch besonders in Hinblick auf die Fantasia über „Ave maris stella“ scheint Rodio eine Korrektur der Polaritäten herbeizuführen, die sich womöglich zu seiner Zeit für seinen Geschmack zu sehr vom kontrapunktischen Substrat zugunsten des kapriziösen Fantasierens entfernt hatte. Für ihn war die kontrapunktische Kunst die entscheidende Basis einer gesunden kompositorischen Handwerkes.

Rodios Wirken fällt in die Zeit, in der sich die Tastenmusik aus den Eigenarten des jeweiligen, technisch inzwischen weit entwickelten Tasteninstrumentes ableitet. Die Freude an virtuosem Stil blitz auch bei ihm immer wieder auf, doch muss virtuose Spielfreude auf der Basis des Kontrapunktes stehen, Kontrapunkt, wie er lebhaft in den Akademien Neapels diskutiert wurde. Rodios Übersiedeln nach Neapel gab ihm das geeignete Umfeld für seine musikalischen Ambitionen; Neapel quasi als Nabel in Hinblick auf die Lage zwischen Palermo und Rom, Spanien und Italien mit den verschiedenen kulturellen Strömungen und Einflüssen. Somit ist Rodios Arbeit nicht anachronistisch, vielmehr folgt sie einer anderen Intention als die Beiträge zur Tastenmusik von Valente (1575), Merulo und A. Gabrieli. Sein „Ave maris stella“ ist ein klingender Beleg ${ }^{54}$. Stilistisch erinnert sein Satz stellenweise an A. de Cabezón und besonders an bestimmte (Cantus firmus-gebundene) Tentos des Portugiesen António Carreiras ${ }^{55}$. Als Schlüsselfigur der portugiesischen Musik ist Carreiras Einfluss auf das Werk spanischer und italienischer Komponisten bisher unbekannt.

Dass Rocco Rodio seine kontrapunktische Satzkunst (1575) mit einem Traktat untermauert (1600), zeigt ihn als pädagogisch klugen und umfassend gebildeten Musiker. Musikalisch kein brodelnder Vulkan mit neuen Eruptionen sondern ein kreativer und umsichtiger Meister der anspruchsvollen und gediegenen kontrapunktischen Satzkunst. Kein feuriger Ausbruch war von ihm zu erwarten; eher versinnbildlicht er ein stabiles Massiv der Beständigkeit und des Fortschritts innerhalb einer Tradition. Er war ein vielseitiger Komponist, der auch Vokalmusik komponierte und publizierte (siehe II. \& V.). Seine weltliche, in Neapel publizierte Vokalmusik gilt bis dato als verschollen. Weitere einzelne Instrumentalstücke, Motetten und Madrigale finden sich in Sammelbänden. Rodios anspruchsvoller Traktat Regole di musica erschien in zwei Neuauflagen. Rodios Arbeiten waren damals auch jenseits süditalienischer Grenzen, besonders in Spanien und Portugal bekannt ${ }^{56} .1963$ schrieb Kastner: „Die überragende Stellung, die Rodio in der südital. Musik der zweiten Hälfte des 16. Jh. einnimmt, ist seitens der modernen Mw. noch nicht in ihrer gesamten Tragweite erfasst worden." Diese Aussage hat heute leider immer noch Gültigkeit.

56. Kastner1963, Sp. 599. Lederer, op.cit. Jessie Ann Owens, The Languages of the Modes: Studies in the History of Polyphonic Modality (Critism and Anlysis of Early Music), New York 2001, S. 15. 